The Astronomical Journal, 115:809-820, 1998 February

(C) 1998. The American Astronomical Society. All rights reserved. Printed in U.S.A.

\title{
THE CARBON-RICH DUST SEQUENCE: INFRARED SPECTRAL CLASSIFICATION OF CARBON STARS
}

\author{
G. C. SLOAN ${ }^{1}$ \\ NASA Ames Research Center, MS 245-6, Moffett Field, CA 94035-1000; sloan@ssa1.arc.nasa.gov \\ I. R. LITTLE-MARENIN \\ Whitin Observatory, Wellesley College, Wellesley, MA 02181; ilittle@lucy.wellesley.edu \\ AND \\ S. D. PRICE \\ Phillips Laboratory/GPOB, 29 Randolph Road, Hanscom AFB, MA 01731-3010; price@plh.af.mil \\ Received 1997 April 28; revised 1997 October 15
}

\begin{abstract}
We have developed a classification system for the infrared spectral emission from carbon stars, using a sample of 96 bright carbon-rich variables associated with the asymptotic giant branch. In addition to the stellar contribution, most spectra include the $11.2 \mu \mathrm{m}$ emission feature from $\mathrm{SiC}$ and either a smooth, cool continuum from amorphous carbon or a secondary emission feature at $9.0 \mu \mathrm{m}$. We have identified a carbon-rich dust sequence along which the amorphous carbon component grows while the $9.0 \mu \mathrm{m}$ feature declines in strength. Along this spectral sequence, the proportion of Mira variables increases, as does the period of variability, the mass-loss rate, and the thickness of the circumstellar shell. Thus the carbon-rich dust sequence appears to be an evolutionary sequence. One class of spectra shows a particularly strong $9.0 \mu \mathrm{m}$ feature, enhanced $\mathrm{C} / \mathrm{O}$ ratio, and several other unusual properties that suggest a different sequence, perhaps related to $\mathrm{J}$ stars.
\end{abstract}

Key words: infrared radiation - stars: carbon

\section{INTRODUCTION}

For most stars, the final stage of internal fusion reactions takes place on the asymptotic giant branch (AGB). On the AGB, a star is burning hydrogen and helium in shells around an inert $\mathrm{C} / \mathrm{O}$ core, and it is experiencing thermal pulses, dredge-ups, long-period pulsations, and mass loss in the surrounding envelope (see, e.g., Iben \& Renzini 1983; Habing 1990; Hearn 1990). During this stage, a star will eject most of its envelope; this material cools as it moves away, condensing into a circumstellar dust shell when the temperature falls below $\sim 1500 \mathrm{~K}$. The composition of the dust reflects the composition of the underlying photosphere, which in turn reflects the amount of processed material dredged to the surface during thermal pulses. Normal $\mathbf{M}$ and $\mathrm{K}$ giants, with more oxygen than carbon in the photosphere, produce oxygen-rich species of dust such as silicates and alumina material. Stars with more processed material at their surface are usually classified as carbon stars, and they are usually surrounded by carbon-rich dust shells.

The low-resolution spectrometer (LRS) aboard IRAS has provided thousands of spectra of circumstellar dust shells. Analysis of these data has led to the identification of several different classes of spectra associated with oxygen-rich dust shells (Vardya, de Jong, \& Willems 1986; Little-Marenin \& Little 1988, 1990; Onaka, de Jong, \& Willems 1989; Simpson 1991) and carbon-rich dust shells (Baron et al. 1987; Papoular 1988; Willems 1988a, 1988b; Willems \& de Jong 1988; Chan \& Kwok 1988, 1990; Goebel, Cheeseman, \& Gerbault 1995).

Little-Marenin \& Little (1988, 1990; hereafter collectively LML) developed a unique approach to infrared spectral classification. Before classifying the spectrum, they first isolated the contribution from the dust by estimating and

\footnotetext{
${ }^{1}$ Current address: School of Physics, University College, Australian Defence Force Academy, Canberra, ACT 2600, Australia.
}

removing the contribution from the star. This step eliminates the contrast between the star and shell as a possible criterion for classification; it led to the identification of several different spectral shapes that must result from differences in the optical efficiencies of the grains or in the optical depth of the shell (or both). Sloan \& Price (1995, hereafter SP1) improved on the LML classification system and demonstrated that all of the infrared spectral classes identified by LML in oxygen-rich dust shells could be ordered along what they called the silicate dust sequence.

SP1 started with a flux-limited sample of 683 AGB variables produced by cross-referencing the General Catalogue of Variable Stars (Kholopov et al. 1985-1988, hereafter GCVS) with the IRAS Point Source Catalog, Version 2 (1988, hereafter PSC). The sample included all four variability classes associated with the AGB: Mira variables, semiregular variables of classes $\mathrm{SRa}$ and $\mathrm{SRb}$, and irregular variables of class $\mathrm{Lb}$. Only sources brighter than $28 \mathrm{Jy}$ at 12 $\mu \mathrm{m}([12]<0.0)$ were considered, in order to eliminate poorquality spectra. The sample included 546 stars of spectral class $\mathrm{M}$ or $\mathrm{K}$ (oxygen-rich photospheres), $21 \mathrm{~S}$ stars (C/O abundance $\sim 1$ ), 96 carbon stars, and 20 stars with no spectral class. SP1 focused on the M and K stars. Sloan \& Price (1997, hereafter SP2) extend the study to include the S stars and variables associated with supergiants (classes SRc and Lc); both of these groups generally exhibit oxygen-rich dust emission. Here, we turn to the 96 carbon stars.

As noted above, the LRS database of spectra from carbon-rich dust shells has received a great deal of attention. Willems (1988a, 1988b) recognized two general classes of infrared spectral emission from carbon-rich dust. One group exhibited an emission feature near $11.2 \mu \mathrm{m}$ and a stellar continuum; the other showed the $11.2 \mu \mathrm{m}$ feature superposed on a much cooler nonstellar continuum. The $11.2 \mu \mathrm{m}$ emission feature almost certainly arises from $\alpha$-SiC (Gilman 1969; Hackwell 1972; Treffers \& Cohen 1974; 
Goebel et al. 1980; Cohen 1984; Pégourié 1987; Groenewegen 1995; Speck, Barlow, \& Skinner 1996, 1997). Amorphous carbon most likely produces the featureless dust continuum seen in most of the cooler spectra. Using a mixture of $\sim 90 \%-95 \%$ amorphous carbon and $\sim 5 \%-10 \%$ $\mathrm{SiC}$, several authors have produced models that fit the emergent spectrum of the prototypical extreme carbon star IRC +10216 (also known as IRAS $09452+1330$ and CW Leo; Martin \& Rogers 1987; Griffin 1990; Sloan \& Egan 1995; Groenewegen 1997). In models for other carbon stars, the amorphous carbon component also usually dominates the SiC component (e.g., Lorenz-Martins \& Lefèvre 1993, 1994; Groenewegen 1995; Lorenz-Martins 1996).

Willems (1988a, 1988b) found that sources with the cooler amorphous carbon component were predominately Mira variables. The group with the warmer stellar continuum usually showed a secondary emission feature $\sim 8.5-9.0$ $\mu \mathrm{m}$ and contained more semiregular and irregular variables. Goebel et al. (1995), in a reanalysis of the AUTOCLASS classification method (Cheeseman et al. 1989; Goebel et al. 1989), suggest that the feature $\sim 8.5-9.0 \mu \mathrm{m}$ arises from a precursor to amorphous carbon.

Our study presents several improvements on previous classification efforts. First, we have corrected the LRS data for the erroneous assumption during calibration that $\alpha$ Tau can be represented by a 10,000 K blackbody (Cohen, Walker, \& Witteborn 1992). This assumption overlooks the fact that all late-type oxygen-rich giants have $\mathrm{SiO}$ absorption bands in the 8-9 $\mu \mathrm{m}$ region, and it introduces an artificial emission feature in this region in all spectra. This artifact peaks at $\sim 8.3 \mu \mathrm{m}$, in the vicinity of the emission component noted by Willems (1988a, 1988b) and Goebel et al. (1995). Before a quantitative analysis of LRS data in this spectral region can be undertaken, this error needs to be corrected. In addition, we will follow the procedure of LML and SP1 and first remove the stellar contribution before classifying the dust spectrum, something other investigators have not done. We also have a very well defined sample of bright sources covering nearly the entire sky, enabling us to draw statistical conclusions and make more thorough comparisons between optical and infrared properties.

In $\S 2$, we develop a self-consistent classification system for spectra from carbon-rich circumstellar dust shells, apply it to the flux-limited sample of carbon-rich AGB variables produced by SP1, and compare our classifications with previous methods. In $\S 3$, we compare the results of our infrared classification with the optical properties of the stars (spectral class, variability class, and period) in an effort to relate the spectral properties of the circumstellar dust with the behavior of the central star.

Our sample excludes both the early and extreme carbon stars because neither group appears in the GCVS. Of the early carbon stars $(\mathrm{C} 0-\mathrm{C} 3)$, only $\sim 10 \%$ pulsate (Mikami 1975). As a consequence, most of these sources probably have not begun to lose significant amounts of mass or to form circumstellar dust shells. While most of the extreme carbon stars are probably long-period Mira variables, our sample includes only three, because most are too heavily enshrouded to be recognized as optical variables.

\section{CLASSIFICATION}

For each source, we obtain a spectrum from the LRS database maintained at the University of Calgary, which we recalibrate using the corrections introduced by Cohen et al.
(1992). To isolate the dust spectrum for classification, we remove the estimated stellar contribution. SP1 describe in detail the nature of spectra from oxygen-rich photospheres, but because the corresponding spectrum from carbon-rich stars remains poorly understood, we simply assume that the star can be approximated by a $2400 \mathrm{~K}$ Planck function. We fit this function to the LRS spectrum over the wavelength range 7.67-8.05 $\mu \mathrm{m}$ (3 pixels) and subtract it. The residual spectrum will include not only the dust spectrum, but also any deviations from a Planck function in the stellar spectrum, which would result from photospheric absorption bands. Figure 1 illustrates some of the extracted spectra.

We assign the extracted spectrum to one of several categories by visual inspection. SP2 describe each at length. Here we are concerned only with three classes: naked $(\mathrm{N})$, silicate or oxygen-rich dust emission (SE), and carbon-rich dust emission (CE). We classify four sources as N, three as SE, and the remaining 89 (the overwhelming majority of the sample) as CE. All 89 of these sources show an emission feature at $\sim 11.2 \mu \mathrm{m}$ typically attributed to $\mathrm{SiC}$ dust.

\subsection{Classes of Carbon-rich Dust Emission}

In the flux ratio plane used by SP1 to define the SE classes, the carbon-rich (CE) and oxygen-rich (SE) dust

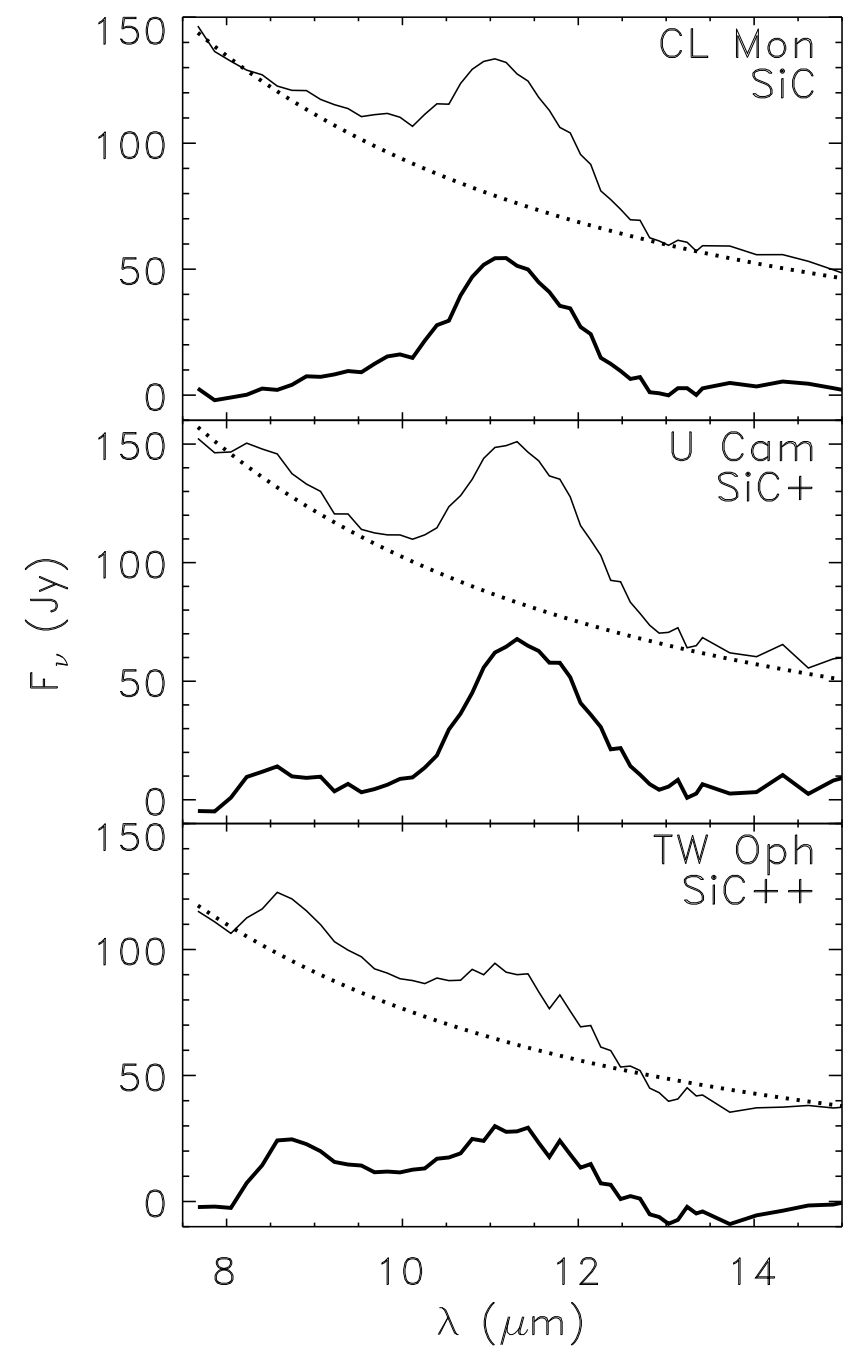

FIG. 1.-Sample dust extractions. In each of the panels, we show the corrected LRS spectrum (thin line), the assumed stellar continuum (dotted line; a $2400 \mathrm{~K}$ Planck function), and the residual dust spectrum (thick line). 
sources do not occupy the same region (Fig. 2). These flux ratios were determined from the extracted spectrum at 10 , 11 , and $12 \mu \mathrm{m}$, using $F_{v}$ units. Compared with the silicate feature, the $\mathrm{SiC}$ feature is stronger at $11 \mu \mathrm{m}$ and much weaker at $10 \mu \mathrm{m}$. As a result, spectra on the flux ratio plot will be shifted away from the silicate dust sequence, up and to the left. The SE sources among the carbon stars are an exception; their strong silicate emission places them right on the silicate dust sequence, as it should. We experimented with different shapes for the stellar continuum (e.g., Planck and Engelke functions) and with different methods of fitting the continuum to the spectrum; the specific positions of the data points moved some, but the carbon-rich dust spectra remain above and to the left of the silicate dust sequence in all cases.

In addition to the $11.2 \mu \mathrm{m}$ emission feature attributed to $\mathrm{SiC}$, many of the spectra show a secondary emission feature at $\sim 8.5-9.0 \mu \mathrm{m}$. The apparent strength of this feature depends on how the continuum is fitted to the spectrum around $8.0 \mu \mathrm{m}$, which may depend on the possible existence

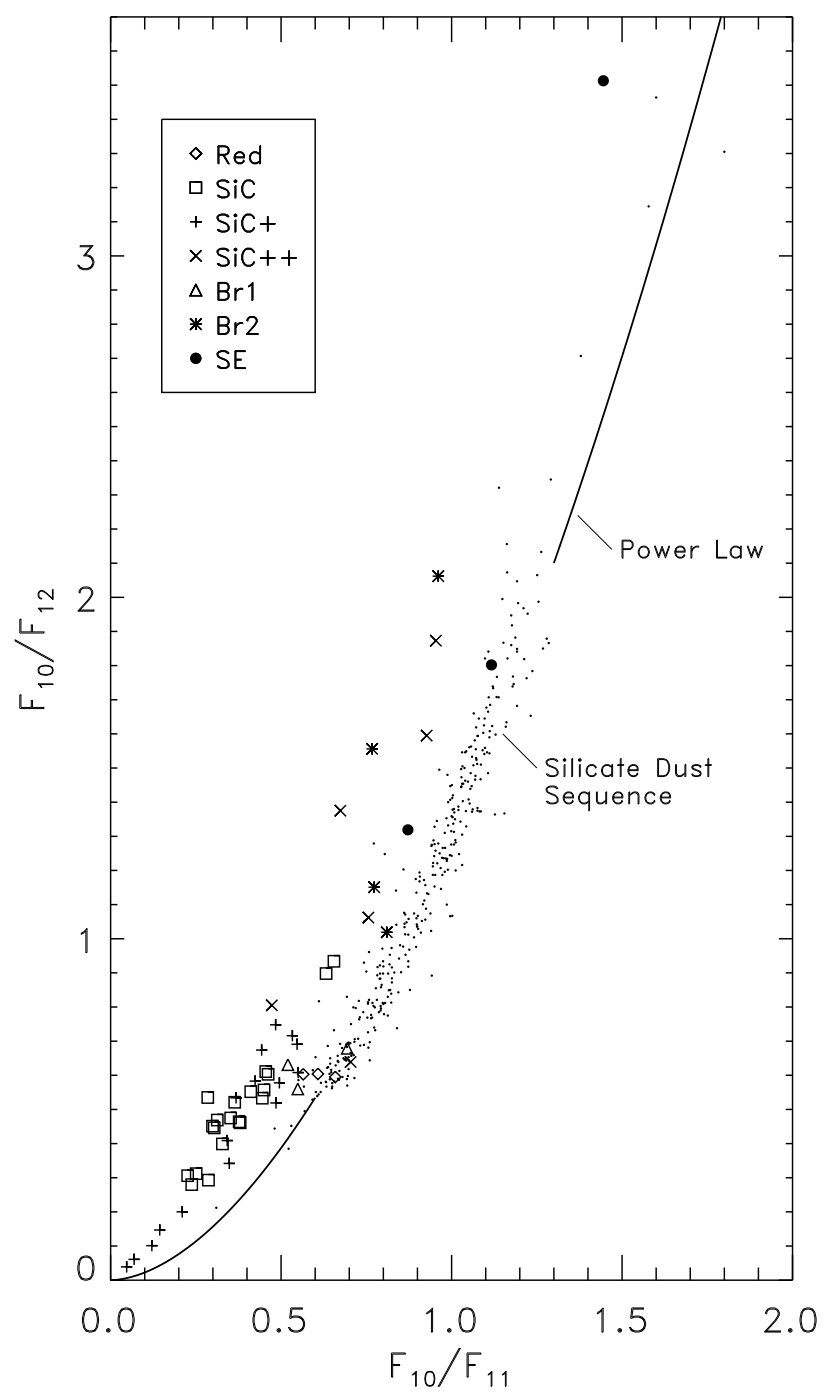

Fig. 2.-The silicate dust sequence compared with the flux ratios for the carbon stars in our sample. The dots represent sources from the oxygen-rich dust sample from SP1; they define the silicate dust sequence. The solid line above and below the silicate dust sequence traces the powerlaw fit to the silicate dust sequence by SP1. The individual flux ratios are in $F_{v}$ units after removing the stellar contribution from the spectrum. of an absorption feature at the edge of the LRS wavelength range $\sim 7.5-7.8 \mu \mathrm{m}$ from $\mathrm{C}_{2} \mathrm{H}_{2}$ or related species like $\mathrm{HCN}$ (Willems 1988a). Whatever the precise origin of the $\sim 9.0$ $\mu \mathrm{m}$ feature, it has proven to be the most significant variable among the carbon-rich dust spectra in our sample. We use its strength as the primary discriminant for classifying the spectra, although we recognize that we may be measuring absorption at shorter wavelengths, or a combination of absorption at $\sim 7.5 \mu \mathrm{m}$ and emission at $\sim 9.0 \mu \mathrm{m}$.

We used the flux ratio $F_{9} / F_{10}$ as an approximate guide to our classification, but we did not base our final classifications on any specific quantities, as done by SP1 and SP2 for oxygen-rich dust, for several reasons. The secondary feature is generally weak compared to the stellar continuum, and its relative strength compared to the $11.2 \mu \mathrm{m}$ feature depends in part on assumptions about the nature of the stellar continuum and how it is fitted to the spectrum. In spectra with particularly low dust-to-star contrasts, noise in the extracted spectrum results in nearly useless flux ratios. Furthermore, roughly $10 \%$ of our sample of carbon stars have spectra that produce normal flux ratios, but which upon visual inspection show shapes that do not match the remainder of the sample (e.g., the Broad spectra defined below).

The majority of carbon-rich dust spectra show either no feature at $\sim 8.5-9.0 \mu \mathrm{m}$, or only a feature of weak or moderate strength. We classify these two groups as " $\mathrm{SiC}$ " or "SiC+," respectively. The primary means of discriminating between them is the shape of the spectrum between 9 and 11 $\mu \mathrm{m}$. A minimum between the features generally results in a classification of $\mathrm{SiC}+$, while a steadily rising dust spectrum from 9 to $11 \mu \mathrm{m}$ results in a classification of SiC. A small number of sources show a much stronger component at $\sim 9.0 \mu \mathrm{m}$, in some cases as strong as or stronger than the $\sim 11.2 \mu$ m feature; we identify these as " $\mathrm{SiC}++$." Three spectra have only one emission feature at $\sim 11.2 \mu \mathrm{m}$, but superposed on a strong continuum increasing in intensity toward longer wavelengths, which we identify as "Red." Finally, several spectra do not fit into this classification scheme. They show either a broad emission feature extending from $\sim 8-9 \mu \mathrm{m}$ to $\sim 12 \mu \mathrm{m}$ ("Broad 1" or "Br1") or an unusual emission feature $\sim 11.2 \mu \mathrm{m}$ with an excess toward longer wavelengths (“ $\mathrm{Br} 2$ ”). All six classes are described in Table 1 and illustrated in Figure 3.

The three Red spectra require some clarification. The prototypical member of this class is the well-studied extreme carbon star IRC +10216 . In the mid-infrared, the dust shells around these sources are optically thick, revealing the limitations of our method of fitting and subtracting a stellar continuum. A more appropriate method would be to use a radiative transfer algorithm to separate the stellar and dust contributions, but this would require assumptions about the nature of the dust. Our method, while quantitatively inadequate for these thick shells, still reveals these sources as having stronger contributions from a red dust continuum than the other classes. This extra dust component is almost certainly amorphous carbon, as discussed in $\S 1$. If our sample included sources too heavily obscured to be in the GCVS, this class would probably be much more heavily populated.

Table 2 presents the classifications for each of our 96 sources, along with other optical and infrared properties that we use in the analysis below. Column (1) gives the name in the PSC while columns (2)-(4) give the variable star iden- 
TABLE 1

Classes of Carbon-rich Dust Emission

\begin{tabular}{crl}
\hline \hline \multicolumn{1}{c}{ Class } & Number & \multicolumn{1}{c}{ Description } \\
\hline Red $\ldots \ldots \ldots$. & 3 & $11.2 \mu$ m feature, strong dust continuum \\
$\mathrm{SiC} \ldots \ldots \ldots \ldots$ & 40 & $11.2 \mu \mathrm{m}$ feature, weak dust continuum \\
$\mathrm{SiC}+\ldots \ldots \ldots$ & 32 & $11.2 \mu \mathrm{m}$ feature, weak feature at $\sim 8.5-9.0 \mu \mathrm{m}$, weak dust continuum \\
$\mathrm{SiC}++\ldots \ldots$ & 6 & Comparable $11.2 \mu \mathrm{m}$ and $\sim 8.5-9.0 \mu \mathrm{m}$ features \\
Broad $1 \ldots \ldots$ & 5 & Unusual 11.2 $\mu \mathrm{m}$ feature profile: short-wavelength excess \\
Broad $2 \ldots \ldots$ & 3 & Unusual $11.2 \mu \mathrm{m}$ feature profile: long-wavelength excess \\
\hline
\end{tabular}

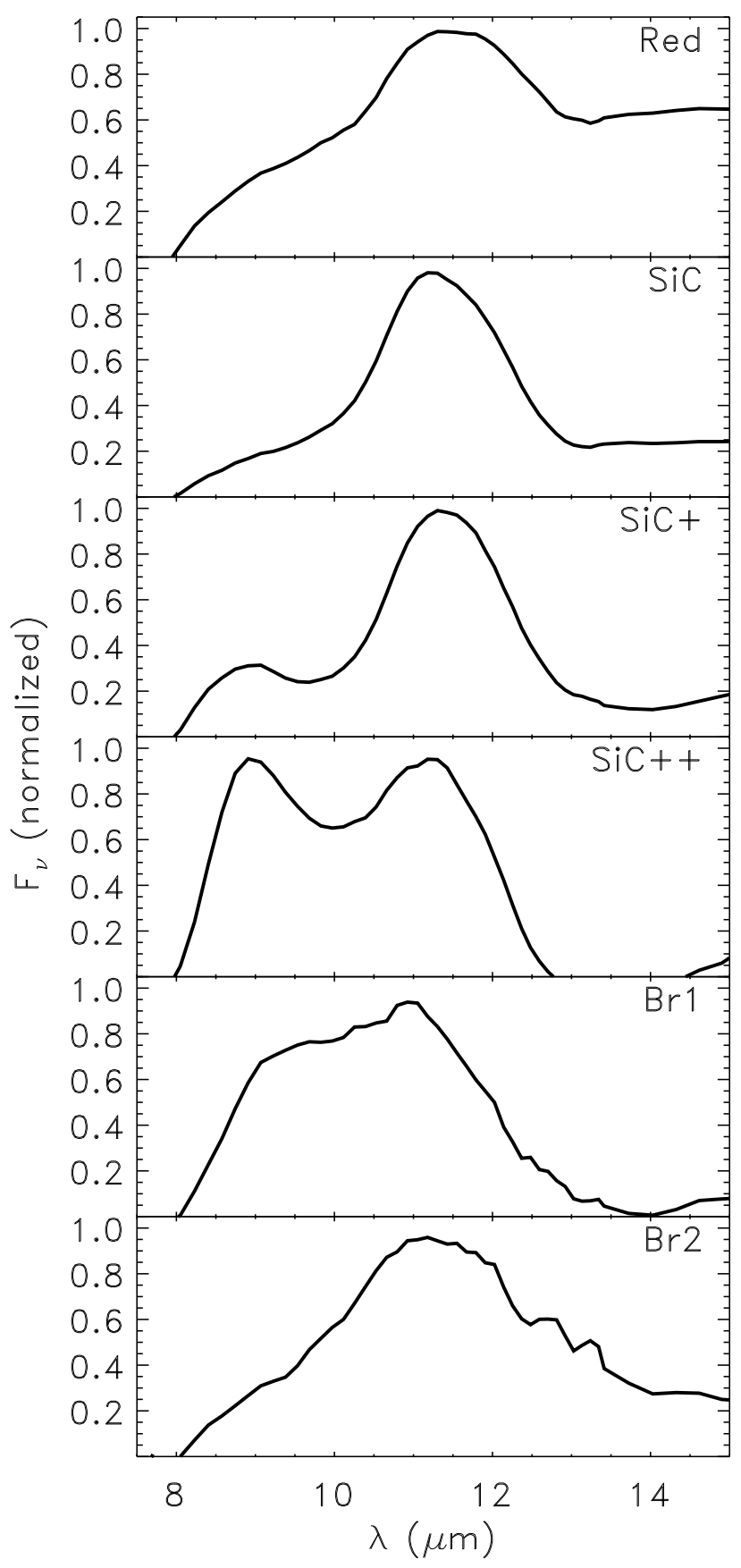

Fig. 3.- Summed dust spectra for each class of carbon-rich dust. Each panel shows the dust spectra for all sources in a class, summed, smoothed with a 3 pixel boxcar, and normalized. tification, classification, and period, respectively. The spectral classes in column (5) are from the GCVS, and the $12 \mu \mathrm{m}$ magnitudes, [12]-[25] colors, and LRS characterizations (cols. [6]-[8]) are from the PSC. We have added a " $[\mathrm{J}]$ " designation to the spectral class of sources identified as $\mathbf{J}$ stars by Lorenz-Martins (1996). AUTOCLASS II classifications in column (9) (Goebel et al. 1995) were provided by Goebel (1995, private communication). Column (10) presents our dust classifications; a colon follows the more uncertain classifications. The dust emission contrasts (DECs) in column (11) are defined by SP1 as the ratio of the summed dust emission to the summed stellar emission from 7.67 to $14.03 \mu \mathrm{m}$.

\subsection{Comparison with the LRS Characterizations}

The Atlas of Low Resolution IRAS Spectra (1986) uses two digits to characterize a spectrum. Carbon-rich dust spectra are generally classified as 41-46 (4n). Oxygen-rich dust spectra are classified as 21-29 $(2 n)$ if in emission and 31-39 (3n) if in absorption. In all three groups the second digit $(n)$ increases with the strength of the dust feature. Spectra with characterizations of 11-19 (1n) are generally misunderstood as naked sources, although spectra in this group could have dust emission that is less than $10 \%$ of the continuum emission at all wavelengths, or less than 5 times the rms noise. For class $1 n$ objects, $n=-2 \beta$, where $\beta$ is defined by the relation $F_{\lambda} \propto \lambda^{\beta}$ between 7 and $13 \mu \mathrm{m}$. Naked stars usually have LRS characterizations of 18 (since $\beta=-4$ on the Rayleigh-Jeans tail).

Table 3 compares our classifications with the LRS characterizations. Of the 79 carbon-rich dust spectra in both classification systems, 65 have LRS characterizations in the range $41-46$. For class $4 n$ sources, $n=10 \ln \left(F_{11.4 \mu \mathrm{m}} / F_{\mathrm{c}}\right)$, where $F_{\mathrm{c}}$ is the interpolated continuum emission at $11.4 \mu \mathrm{m}$. (See the discussion in the LRS atlas for more details.) Thus, in the LRS characterizations the contrast between the dust and star is the primary discriminant among carbon-rich dust spectra.

In our system, we remove the stellar contribution before classifying the dust spectrum, allowing us to treat the shape of the emission profile and the contrast of the shell as independent variables. As Table 3 shows, the ratio of $\mathrm{SiC}$ spectra to $\mathrm{SiC}+$ and other carbon-rich emission (CE) classes increases steadily from LRS characterizations 41 to 46. This trend identifies the tendency of $\mathrm{SiC}$ spectra to have stronger dust emission with respect to the continuum (as defined by the LRS characterizations) than the other CE classes.

Figure 4 compares the distribution of dust emission con- 
TABLE 2

InFraRed SpeCtral Classifications of Carbon Stars

\begin{tabular}{|c|c|c|c|c|c|c|c|c|c|c|}
\hline $\begin{array}{l}\text { PSC } \\
(1)\end{array}$ & $\begin{array}{l}\text { Name } \\
(2)\end{array}$ & $\begin{array}{l}\text { Variability } \\
\text { (3) }\end{array}$ & $\begin{array}{c}\text { Period } \\
\text { (days) } \\
\text { (4) }\end{array}$ & $\begin{array}{l}\text { Spectral Class } \\
\text { (5) }\end{array}$ & $\begin{array}{l}{[12]} \\
(6)\end{array}$ & $\begin{array}{c}{[12]-[25]} \\
(7)\end{array}$ & $\begin{array}{l}\text { LRS } \\
(8)\end{array}$ & $\begin{array}{l}\mathrm{AC} \text { II } \\
(9)\end{array}$ & $\begin{array}{l}\text { Dust } \\
(10)\end{array}$ & $\begin{array}{c}\text { DEC } \\
(11)\end{array}$ \\
\hline $00036+6947 .$. & OR Cep & $\mathrm{Lb}$ & & $\mathrm{C}$ & -0.28 & 0.14 & 44 & 3 & $\mathrm{SiC}+$ & 0.22 \\
\hline $00172+4425 \ldots \ldots$ & VX And & $\mathrm{SRa}$ & 369.00 & $\mathrm{C} 4,5$ (N7) [J] & -0.68 & 0.22 & 24 & 3 & $\mathrm{SiC}++$ & 0.33 \\
\hline $01080+5327 \ldots \ldots$ & HV Cas & Mira & 527.00 & $\mathrm{C} 4,3-\mathrm{C} 5,4 \mathrm{e}(\mathrm{Ne})$ & -0.93 & 0.44 & 44 & 0 & $\mathrm{SiC}$ & 0.33 \\
\hline $01133+2530 \ldots \ldots$ & Z Psc & $\mathrm{SRb}$ & 144.00 & $\mathrm{C} 7,2$ (N0) & -0.18 & 0.37 & 22 & 3 & $\mathrm{SiC}+:$ & 0.14 \\
\hline $01246-3248 \ldots \ldots$ & R Scl & $\mathrm{SRb}$ & 370.00 & $\mathrm{C6}, 5 \mathrm{ea}(\mathrm{Np})$ & -1.89 & 0.82 & & .. & $\mathrm{SiC}++$ & 0.30 \\
\hline $02270-2619 \ldots \ldots$ & R For & Mira & 388.73 & $\mathrm{C} 4,3 \mathrm{e}(\mathrm{Ne})$ & -2.38 & 0.24 & 43 & 0 & $\mathrm{SiC}$ & 0.19 \\
\hline $03075+5742 \ldots \ldots$ & V623 Cas & Lb: & & $\mathrm{C} 4,5 \mathrm{~J}$ (R5) & -0.32 & -0.05 & 31 & 6 & $\mathrm{~N}:$ & -0.07 \\
\hline $03112-5730 \ldots \ldots$ & TW Hor & $\mathrm{SRb}$ & 158.00 & $\mathrm{C} 7,2$ (N0) & -1.30 & 0.53 & 43 & 0 & $\mathrm{SiC}$ & 0.19 \\
\hline $03229+4721 \ldots \ldots$ & V384 Per & Mira & 535.00 & $\mathrm{C}(\mathrm{N})$ & -3.19 & 0.48 & 44 & 0 & $\mathrm{SiC}$ & 0.36 \\
\hline $03374+6229 \ldots \ldots$ & U Cam & $\mathrm{SRb}$ & $\ldots$ & C3,9-C6,4e (N5) & -1.58 & 0.38 & 45 & 0 & $\mathrm{SiC}+$ & 0.20 \\
\hline $03488+3943 \ldots \ldots$ & V414 Per & $\mathrm{Lb}$ : & $\ldots$ & $\mathrm{C}$ & -1.74 & 0.16 & 42 & .. & $\mathrm{SiC}$ & 0.13 \\
\hline $04262+3945 \ldots \ldots$ & GI Per & $\mathrm{SRb}$ & 400.00 & $\mathrm{C} 7,4(\mathrm{~N})$ & -0.52 & 0.31 & 45 & 4 & SiC: & 0.39 \\
\hline $04284+1732 \ldots \ldots$ & V718 Tau & Mira & 405.00 & $\mathrm{C} 2: \mathrm{e}(\mathrm{Ne})$ & -1.01 & -0.01 & 45 & 0 & $\mathrm{SiC}$ & 0.15 \\
\hline $04459+6804 \ldots \ldots$ & ST Cam & $\mathrm{SRb}$ & 300.00 & C5,4 (N5) & -1.31 & 0.17 & 42 & 3 & $\mathrm{SiC}+:$ & 0.11 \\
\hline $04483+2826 \ldots \ldots$ & TT Tau & $\mathrm{SRb}$ & 166.50 & $\mathrm{C} 4,2-\mathrm{C} 7,4(\mathrm{~N} 3)$ & -0.17 & 0.33 & 22 & 9 & $\mathrm{SiC}+:$ & 0.19 \\
\hline $04573-1452 \ldots \ldots$ & R Lep & Mira & 427.07 & $\mathrm{C} 7,6 \mathrm{e}(\mathrm{N} 6 \mathrm{e})$ & -2.82 & 0.28 & 45 & 0 & $\mathrm{SiC}$ & 0.23 \\
\hline $04595+5033 \ldots \ldots$ & EL Aur & $\mathrm{Lb}$ & & C5,4 (N3) & -0.18 & 0.35 & 42 & 3 & $\mathrm{SiC}+$ & 0.12 \\
\hline $05028+0106 \ldots \ldots$ & W Ori & $\mathrm{SRb}$ & 212.00 & C5,4 (N5) & -2.03 & 0.18 & 44 & 0 & $\mathrm{SiC}+$ & 0.27 \\
\hline $05131+1155 \ldots \ldots$ & V431 Ori & $\mathrm{SRb}$ & 122.00 & $\mathrm{C} 5,5(\mathrm{~N})$ & -0.25 & 0.21 & 44 & 3 & $\mathrm{SiC}+$ & 0.16 \\
\hline $05149+3511 \ldots \ldots$ & DY Aur & Mira & 473.84 & $\mathrm{C}(\mathrm{R})$ & -0.38 & 0.20 & 43 & 3 & $\mathrm{SiC}$ & 0.13 \\
\hline $05185+3227 \ldots \ldots$ & UV Aur & Mira & 394.42 & C6,2-C8,2Jep (Ne) & -0.97 & 0.24 & 43 & 0 & $\mathrm{SiC}+$ & 0.32 \\
\hline $05418-4628 \ldots \ldots$ & W Pic & $\mathrm{Lb}$ & (2) & $\mathrm{C}(\mathrm{N})$ & -0.75 & 0.25 & 41 & 0 & $\mathrm{SiC}++$ : & 0.19 \\
\hline $05421+2424 \ldots \ldots$ & TU Tau & $\mathrm{SRb}$ & 190.00 & $\mathrm{C} 5,4(\mathrm{~N} 3)+\mathrm{A} 2 \mathrm{III}-\mathrm{V}$ & -0.24 & 0.10 & 42 & 3 & $\mathrm{SiC}:$ & -0.01 \\
\hline $05426+2040 \ldots \ldots$ & Y Tau & $\mathrm{SRb}$ & 241.50 & C6.5,4e (N3) & -1.77 & 0.43 & 45 & 0 & $\mathrm{SiC}$ & 0.25 \\
\hline $05576+3940 \ldots \ldots$ & AZ Aur & Mira & 415.92 & $\mathrm{C} 7,1 \mathrm{e}-\mathrm{C} 8,2-3(\mathrm{~N} 0 \mathrm{e})$ & -0.61 & 0.21 & 42 & 0 & $\mathrm{Br} 2$ & 0.31 \\
\hline $06077+2601 \ldots \ldots$ & TU Gem & $\mathrm{SRb}$ & 230.00 & C6,4 (N3) & -0.99 & 0.23 & & .. & $\mathrm{SiC}$ & 0.25 \\
\hline $06225+1445 \ldots \ldots$ & BL Ori & $\mathrm{Lb}$ & $\ldots$ & $\mathrm{C} 6,3(\mathrm{Nb}, \mathrm{Tc})$ & -0.49 & 0.30 & 16 & 3 & $\mathrm{Br} 2$ & 0.12 \\
\hline $06226-0905 \ldots \ldots$ & V636 Mon & Mira: & $\ldots$ & $\mathrm{C}$ & -1.61 & 0.32 & 43 & 0 & $\mathrm{SiC}+$ & 0.26 \\
\hline $06315+1606 \ldots \ldots$ & CR Gem & $\mathrm{Lb}$ & $\ldots$ & $\mathrm{C} 8,3 \mathrm{e}(\mathrm{N})$ & -0.36 & 0.60 & 42 & 1 & $\mathrm{Br} 2$ & 0.41 \\
\hline $06331+3829 \ldots \ldots$ & UU Aur & $\mathrm{SRb}$ & 234.00 & $\mathrm{C} 5,3-\mathrm{C} 7,4$ (N3) & -2.28 & 0.28 & 43 & 0 & $\mathrm{SiC}$ & 0.15 \\
\hline $06504-1206 \ldots \ldots$ & GP CMa & Lb: & $\ldots$ & Cea: & -0.72 & 0.25 & 45 & 0 & $\mathrm{SiC}$ & 0.24 \\
\hline $06528-4218 \ldots \ldots$ & NP Pup & $\mathrm{Lb}$ & & $\mathrm{C} 7,2$ (N0) & -0.26 & 0.44 & 42 & 3 & SE5: & 0.13 \\
\hline $06529+0626 \ldots \ldots$ & CL Mon & Mira & 497.15 & C6,3e (N6e) & -1.51 & 0.12 & 44 & 0 & $\mathrm{SiC}$ & 0.15 \\
\hline $06556+0614 \ldots \ldots$ & RV Mon & $\mathrm{SRb}$ & 131.50 & $\mathrm{C} 4,4-\mathrm{C} 6,2(\mathrm{Nb} / \mathrm{R} 9)$ & -0.10 & 0.16 & 43 & 3 & $\mathrm{SiC}$ & 0.10 \\
\hline $07045-0728 \ldots \ldots$ & RY Mon & $\mathrm{SRa}$ & 455.70 & $\mathrm{C} 5,5-\mathrm{C} 7,4:(\mathrm{N} 5 / \mathrm{R})$ & -0.79 & 0.21 & 45 & 3 & $\mathrm{SiC}+$ & 0.14 \\
\hline $07057-1150 \ldots \ldots$ & $\mathrm{W} \mathrm{CMa}$ & $\mathrm{Lb}$ & & $\mathrm{C} 6,3(\mathrm{~N})$ & -0.35 & 0.62 & 42 & 3 & $\mathrm{SiC}+:$ & 0.15 \\
\hline $07065-7256 \ldots \ldots$ & R Vol & Mira & 453.60 & $\mathrm{Ce}(\mathrm{N})$ & -2.14 & 0.48 & 45 & 0 & $\mathrm{SiC}$ & 0.30 \\
\hline $07179+2505 \ldots \ldots$ & BM Gem & $\mathrm{SRb}$ & 286.00 & $\mathrm{C} 5,4 \mathrm{~J}(\mathrm{Nb})$ & -0.23 & 0.93 & 29 & 11 & SE8 & 1.76 \\
\hline $07487-0229 \ldots \ldots$ & V633 Mon & Mira: & & $\mathrm{C}$ & -0.49 & 0.34 & 42 & 3 & $\mathrm{SiC}$ & 0.16 \\
\hline $08174+0255 \ldots \ldots$ & RY Hya & $\mathrm{SRb}$ & 529.00 & $\mathrm{C} 6,4 \mathrm{e}(\mathrm{Nb})$ & -0.05 & 0.20 & 45 & 4 & $\mathrm{SiC}+$ & 0.37 \\
\hline $08525+1725 \ldots \ldots$ & $\mathrm{X} \mathrm{Cnc}$ & $\mathrm{SRb}$ & 195.00 & $\mathrm{C} 5,4(\mathrm{~N} 3)$ & -1.26 & 0.19 & 42 & 3 & $\mathrm{SiC}+$ & 0.16 \\
\hline $08538+2002 \ldots \ldots$ & $\mathrm{T} \mathrm{Cnc}$ & $\mathrm{SRb}$ & 482.00 & $\mathrm{C} 3,8-\mathrm{C} 5,5$ (R6-N6) & -0.84 & 0.13 & 23 & 3 & $\mathrm{SiC}++$ & 0.18 \\
\hline $09112-2311 \ldots \ldots$ & IQ Hya & Mira & & $\mathrm{C} 3,2(\mathrm{Ne})$ & -0.23 & 0.19 & 45 & 0 & $\mathrm{SiC}$ & 0.29 \\
\hline $09452+1330 \ldots \ldots$ & CW Leo & Mira & 630.00 & $\mathrm{C} 9,5$ & -8.06 & 0.78 & 43 & 5 & Red & 0.61 \\
\hline $10131+3049 \ldots \ldots$ & RW LMi & $\mathrm{SRa}$ & 640.00 & $\mathrm{C} 4,3 \mathrm{ev}$ & -5.17 & 0.47 & 04 & 3 & $\mathrm{SiC}:$ & 0.15 \\
\hline $10249-2517 \ldots \ldots$ & CZ Hya & Mira & 442.00 & $\mathrm{Ce}(\mathrm{Ne})$ & -0.67 & 0.50 & 46 & 0 & $\mathrm{SiC}$ & 0.33 \\
\hline $10329-3918 \ldots \ldots$ & U Ant & $\mathrm{Lb}$ & & $\mathrm{C} 5,3(\mathrm{Nb})$ & -1.93 & 0.13 & 21 & 3 & $\mathrm{SiC}+:$ & 0.07 \\
\hline $10350-1307 \ldots \ldots$ & U Hya & $\mathrm{SRb}$ & 450.00 & $\mathrm{C} 6.5,3$ (N2) (Tc) & -2.15 & 0.43 & & .. & $\mathrm{SiC}$ & 0.14 \\
\hline $10368-6033 \ldots \ldots$ & TV Car & $\mathrm{Lb}$ : & $\ldots$ & $\mathrm{C}$ & -0.19 & 0.39 & 45 & 4 & $\mathrm{SiC}$ & 0.36 \\
\hline $10416+6740 \ldots \ldots$ & VY UMa & $\mathrm{Lb}$ & & C6,3 (N0) & -0.66 & 0.14 & 42 & 3 & $\mathrm{SiC}$ & 0.06 \\
\hline $10491-2059 \ldots \ldots$ & V Hya & $\mathrm{SRa}$ & 530.70 & $\mathrm{C} 6,3 \mathrm{e}-\mathrm{C} 7,5 \mathrm{e}(\mathrm{N} 6 \mathrm{e})$ & -3.98 & 0.61 & $\ldots$ & .. & Red & 0.36 \\
\hline $12226+0102 \ldots \ldots$ & SS Vir & $\mathrm{SRa}$ & 364.14 & $\mathrm{C} 6,3 \mathrm{e}(\mathrm{Ne})$ & -1.28 & 0.30 & 04 & 3 & $\mathrm{Br} 1$ & 0.40 \\
\hline $12427+4542 \ldots \ldots$ & Y CVn & $\mathrm{SRb}$ & 157.00 & $\mathrm{C} 5,4 \mathrm{~J}(\mathrm{~N} 3)$ & -2.47 & 0.07 & 42 & 3 & $\mathrm{SiC}+:$ & 0.07 \\
\hline $12447+0425 \ldots \ldots$ & RU Vir & Mira & 433.20 & C8,1e (R3ep) & -2.27 & 0.26 & 44 & 0 & $\mathrm{SiC}$ & 0.36 \\
\hline $12465-6129 \ldots \ldots$ & RX Cru & Lb: & & Cea (N:) & -0.53 & 0.19 & 44 & 3 & $\mathrm{SiC}+$ & 0.12 \\
\hline $12544+6615 \ldots \ldots$ & RY Dra & SRb: & 200.00 & $\mathrm{C} 4,5 \mathrm{~J}$ (N4p) & -1.46 & 0.20 & 41 & 3 & $\mathrm{SiC}+$ & 0.08 \\
\hline $13343-5613 \ldots \ldots$ & RV Cen & Mira & 446.00 & N3e & -0.27 & 0.41 & 25 & 6 & $\mathrm{Br} 1$ & 0.41 \\
\hline $15094-6953 \ldots \ldots$ & X Tra & $\mathrm{Lb}$ & $\ldots$ & $\mathrm{C} 5,5(\mathrm{Nb})$ & -2.13 & 0.19 & 42 & 3 & $\mathrm{SiC}+$ & 0.10 \\
\hline $15219-7545 \ldots \ldots$ & U Aps & $\mathrm{Lb}$ & & $\mathrm{C}(\mathrm{Nb})$ & -0.00 & 0.10 & 44 & 3 & $\mathrm{SiC}+$ & 0.06 \\
\hline $15477+3943 \ldots \ldots$ & $\mathrm{V} \mathrm{CrB}$ & Mira & 357.63 & $\mathrm{C} 6,2 \mathrm{e}(\mathrm{N} 2 \mathrm{e})$ & -1.42 & 0.28 & $\ldots$ & .. & $\mathrm{SiC}$ & 0.30 \\
\hline $16239-1218 \ldots \ldots$ & V Oph & Mira & 297.21 & $\mathrm{C} 5,2-\mathrm{C} 7,4 \mathrm{e}(\mathrm{N} 3 \mathrm{e})$ & -0.03 & 0.20 & 43 & 3 & $\mathrm{SiC}+$ & 0.17 \\
\hline $17103-3551 \ldots \ldots$ & V617 Sco & Mira & 523.60 & $\mathrm{C}$ & -0.82 & 0.78 & 44 & 0 & $\mathrm{SiC}$ & 0.29 \\
\hline $17267-1926 \ldots \ldots$ & TW Oph & $\mathrm{SRb}$ & 185.00 & $\mathrm{C} 5,5(\mathrm{Nb})$ & -1.33 & 0.13 & 43 & 3 & $\mathrm{SiC}++$ & 0.16 \\
\hline $17389-5742 \ldots \ldots$ & V Pav & $\mathrm{SRb}$ & 225.40 & $\mathrm{C} 6,4(\mathrm{Nb})$ & -1.52 & 0.42 & 45 & 0 & $\mathrm{SiC}+$ & 0.39 \\
\hline $17556+5813 \ldots \ldots$ & T Dra & Mira & 421.62 & $\mathrm{C} 6,2 \mathrm{e}-\mathrm{C} 8,3 \mathrm{e}(\mathrm{NOe})$ & -2.11 & 0.37 & 45 & 0 & $\mathrm{SiC}$ & 0.40 \\
\hline $18040-0941 \ldots \ldots$ & FX Ser & Lb: & & $\mathrm{C}$ & -2.19 & 0.56 & 44 & 0 & $\mathrm{SiC}$ & 0.21 \\
\hline $18073-2652 \ldots \ldots$ & V1280 Sgr & Mira & 523.00 & $\mathrm{C} 6-7,3(\mathrm{~N})$ & -1.25 & 0.33 & 45 & 0 & $\mathrm{SiC}$ & 0.21 \\
\hline $18155-1327 \ldots \ldots$ & ES Ser & $\mathrm{Lb}$ & $\ldots$ & $\mathrm{C}(\mathrm{N})$ & -0.12 & 0.20 & & .. & $\mathrm{SiC}+:$ & -0.11 \\
\hline $18306+3657 \ldots \ldots$ & T Lyr & $\mathrm{Lb}$ & $\ldots$ & $\mathrm{C} 6,5$ (R6) $[\mathrm{J}]$ & -1.28 & 0.38 & 04 & 3 & $\mathrm{SiC}++$ & 0.30 \\
\hline $18476-0758 \ldots \ldots$ & S Sct & $\mathrm{SRb}$ & 148.00 & $\mathrm{C} 6,4(\mathrm{~N} 3)$ & -0.91 & 0.12 & & $\ldots$ & $\mathrm{N}$ & -0.05 \\
\hline $19017-0545 \ldots \ldots$ & V Aql & $\mathrm{SRb}$ & 353.00 & $\mathrm{C} 5,4-\mathrm{C} 6,4$ (N6) & -1.81 & 0.07 & 42 & 3 & $\mathrm{SiC}+$ & 0.10 \\
\hline
\end{tabular}


TABLE 2-Continued

\begin{tabular}{|c|c|c|c|c|c|c|c|c|c|c|}
\hline $\begin{array}{l}\text { PSC } \\
(1)\end{array}$ & $\begin{array}{l}\text { Name } \\
(2)\end{array}$ & $\begin{array}{l}\text { Variability } \\
\text { (3) }\end{array}$ & $\begin{array}{l}\text { Period } \\
\text { (days) } \\
\text { (4) }\end{array}$ & $\begin{array}{c}\text { Spectral Class } \\
\text { (5) }\end{array}$ & $\begin{array}{c}{[12]} \\
(6)\end{array}$ & $\begin{array}{c}{[12]-[25]} \\
(7)\end{array}$ & $\begin{array}{c}\text { LRS } \\
(8)\end{array}$ & $\begin{array}{c}\mathrm{AC} \text { II } \\
(9)\end{array}$ & $\begin{array}{l}\text { Dust } \\
(10)\end{array}$ & $\begin{array}{c}\text { DEC } \\
(11)\end{array}$ \\
\hline $19162-1600 \ldots \ldots$ & V1942 Sgr & $\mathrm{Lb}$ & & C6,4 (N2/R8) & -0.06 & 0.10 & 18 & 3 & $\mathrm{~N}$ & -0.06 \\
\hline $19276-0056 \ldots \ldots$ & V374 Aql & $\mathrm{SRa}$ & 456.50 & $\mathrm{C} 7,3(\mathrm{Ne})$ & -0.68 & 0.63 & 45 & 0 & $\mathrm{SiC}$ & 0.30 \\
\hline $19314-1629 \ldots \ldots$ & AQ Sgr & $\mathrm{SRb}$ & 199.60 & C7,4 (N3) & -0.75 & 0.36 & 43 & 3 & $\mathrm{SiC}+$ & 0.13 \\
\hline $19555+4407 \ldots \ldots$ & AX Cyg & $\mathrm{Lb}$ & 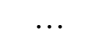 & $\mathrm{C} 4,5$ (N6) & -0.26 & 0.13 & 42 & 3 & $\mathrm{SiC}+:$ & 0.00 \\
\hline $20396+4757 \ldots \ldots$ & V Cyg & Mira & 421.27 & $\mathrm{C} 5,3 \mathrm{e}-\mathrm{C} 7,4 \mathrm{e}(\mathrm{Npe})$ & -3.43 & 0.43 & 44 & 0 & $\mathrm{SiC}$ & 0.25 \\
\hline $21032-0024 \ldots \ldots$ & RV Aqr & Mira & 453.50 & $\mathrm{C} 6-7,2-4(\mathrm{Ne})$ & -2.59 & 0.50 & 45 & 0 & $\mathrm{SiC}$ & 0.31 \\
\hline $21035+5136 \ldots \ldots$ & V1549 Cyg & $\mathrm{Lb}$ & & $\mathrm{C} 6,4$ & -2.38 & 0.64 & 44 & 0 & $\mathrm{SiC}+$ & 0.23 \\
\hline $21168-4514 \ldots \ldots$ & $\mathrm{T}$ Ind & $\mathrm{SRb}$ & 320.00 & $\mathrm{C} 7,2(\mathrm{Na})$ & -0.56 & 0.18 & 17 & 3 & $\mathrm{SiC}:$ & 0.03 \\
\hline $21197-6956 \ldots \ldots$ & Y Pav & $\mathrm{SRb}$ & 233.30 & C7,3 (N0) & -1.02 & 0.48 & 22 & 3 & $\mathrm{Br} 1$ & 0.20 \\
\hline $21232+5705 \ldots \ldots$ & V345 Cep & $\mathrm{SRa}$ & 417.70 & $\mathrm{C}$ & -0.49 & 0.55 & 44 & 0 & $\mathrm{SiC}$ & 0.37 \\
\hline $21399+3516 \ldots \ldots$ & V460 Cyg & $\mathrm{SRb}$ & 180.00 & $\mathrm{C} 6,4$ (N1) & -1.07 & 0.18 & 42 & 3 & $\mathrm{SiC}+:$ & 0.10 \\
\hline $21412+3747 \ldots \ldots$ & RV Cyg & $\mathrm{SRb}$ & 263.00 & $\mathrm{C} 6,4 \mathrm{e}(\mathrm{N} 5)$ & -1.40 & 0.28 & $\ldots$ & $\ldots$ & $\mathrm{SiC}+$ & 0.12 \\
\hline $21440+7324 \ldots \ldots$ & PQ Cep & Mira & $\ldots$ & $\mathrm{C} 6-, 3 \mathrm{e}(\mathrm{N})$ & -1.72 & 0.26 & 44 & 0 & $\mathrm{SiC}+$ & 0.29 \\
\hline $23320+4316 \ldots \ldots$ & LP And & Mira: & $\ldots$ & $\mathrm{C} 8,3.5 \mathrm{e}$ & -3.83 & 0.78 & 42 & 1 & Red & 0.48 \\
\hline $23438+0312$ & TX Psc & $\mathrm{Lb}$ & $\ldots$ & C7,2 (N0) (Tc) & -1.90 & 0.03 & $\ldots$ & $\ldots$ & $\mathrm{N}$ & 0.07 \\
\hline $23587+6004 \ldots \ldots$ & WZ Cas & $\mathrm{SRb}$ & 186.00 & C9,2J Li (N1p) & -0.48 & 0.28 & 23 & 3 & $\mathrm{Br} 1$ & 0.18 \\
\hline
\end{tabular}

trasts for each class of carbon-rich dust emission. The Red spectra show the most dust emission and, thus, have the thickest shells (either optically or geometrically, or both). $\mathrm{SiC}$ spectra show weaker contrasts, and $\mathrm{SiC}+$ spectra are weaker still (although the overlap between these two groups is large). The limited number of spectra of classes $\mathrm{Br} 1, \mathrm{Br}$, and $\mathrm{SiC}++$ prevent any definite conclusions, although the contrast distribution of the $\mathrm{SiC}++$ sources looks similar to the $\mathrm{SiC}$ spectra.

Fourteen of the carbon-rich spectra fall outside of the 41-46 range, illustrating the limitations of the LRS characterizations. Nine CE spectra have been confused for weak SE sources (21-25), especially the $\mathrm{Br} 1$ sources; two have emission too low to characterize (16-17), and three more are characterized as 04 (implying unusual carbon-rich emission).

\subsection{Comparison with the AUTOCLASS System}

The AUTOCLASS system (Goebel et al. 1989; Cheeseman et al. 1989) applied a Bayesian algorithm to classify the entire LRS database. Goebel et al. (1995) reexamined the carbon stars alone, using a similar technique to assign each spectrum to one of 12 classes, identified as class 0 through 11 . About $75 \%$ of our sample falls into either class 0 or 3 (Table 4). Class 0 corresponds to our $\mathrm{SiC}$ class, while class 3 corresponds to our $\mathrm{SiC}+$ class. However, class 3 also contains many other sources that we are able to distinguish as members of separate classes. Only a handful of sources in class 1 or 5 appear in our sample, because these classes correspond to the more heavily enshrouded stars that do not appear in the GCVS. Class 11 corresponds to SE emission, i.e., the silicate/carbon stars. The majority of the remaining classes probably represent low signal-tonoise ratio analogs of the brighter AUTOCLASS classes discussed above (as can be seen by examining the mean $12 \mu$ m magnitudes in Goebel et al. 1995).

\subsection{Silicate/Carbon Stars}

Our sample includes two well-known silicate/carbon stars: BM Gem and V778 Cyg. Both, as illustrated in Figure 5 , show very strong classic silicate emission (Sil in the LML system, SE7 or SE8 in the SP1 system). The comparison spectra were produced by SP2, who summed all spectra of each SE index in the oxygen-rich AGB sample together.

TABLE 3

COMPARISON WITH THE LRS CHARACTERIZATIONS

\begin{tabular}{|c|c|c|c|c|c|c|c|c|c|c|c|c|}
\hline \multirow[b]{2}{*}{ Class } & \multicolumn{12}{|c|}{ LRS CHARACTERIZATION } \\
\hline & None & 04 & $16-18$ & $21-25$ & 29 & 31 & 41 & 42 & 43 & 44 & 45 & 46 \\
\hline Red. & 1 & $\ldots$ & $\ldots$ & $\ldots$ & $\ldots$ & $\ldots$ & $\ldots$ & 1 & 1 & & . & ... \\
\hline $\mathrm{SiC} \ldots \ldots \ldots$ & 4 & 1 & 1 & $\ldots$ & $\ldots$ & $\ldots$ & $\ldots$ & 4 & 5 & 11 & 13 & 1 \\
\hline $\mathrm{SiC}+\ldots \ldots \ldots$ & 4 & $\ldots$ & .. & 3 & $\ldots$ & $\cdots$ & 1 & 9 & 4 & 7 & 4 & ... \\
\hline $\mathrm{SiC}++\ldots \ldots$ & 1 & 1 & $\ldots$ & 2 & $\ldots$ & $\ldots$ & 1 & $\ldots$ & 1 & $\ldots$ & $\ldots$ & ... \\
\hline Br1 $\ldots \ldots \ldots$ & $\ldots$ & 1 & $\ldots$ & 4 & $\ldots$ & $\ldots$ & $\ldots$ & $\ldots$ & $\ldots$ & $\ldots$ & $\ldots$ & $\ldots$ \\
\hline $\mathrm{Br} 2 \ldots \ldots \ldots$ & $\ldots$ & $\ldots$ & 1 & .. & $\ldots$ & .. & $\ldots$ & 2 & .. & $\ldots$ & $\ldots$ & $\ldots$ \\
\hline SE $\ldots \ldots \ldots \ldots$ & $\ldots$ & $\ldots$ & $\ldots$ & $\ldots$ & 2 & $\ldots$ & $\ldots$ & 1 & $\ldots$ & $\ldots$ & $\ldots$ & $\ldots$ \\
\hline $\mathrm{N} \ldots \ldots \ldots \ldots$ & 2 & $\ldots$ & 1 & $\ldots$ & $\ldots$ & 1 & $\ldots$ & $\ldots$ & $\ldots$ & $\ldots$ & $\ldots$ & $\ldots$ \\
\hline Total....... & 12 & 3 & 3 & 9 & 2 & 1 & 2 & 17 & 11 & 18 & 17 & 1 \\
\hline
\end{tabular}




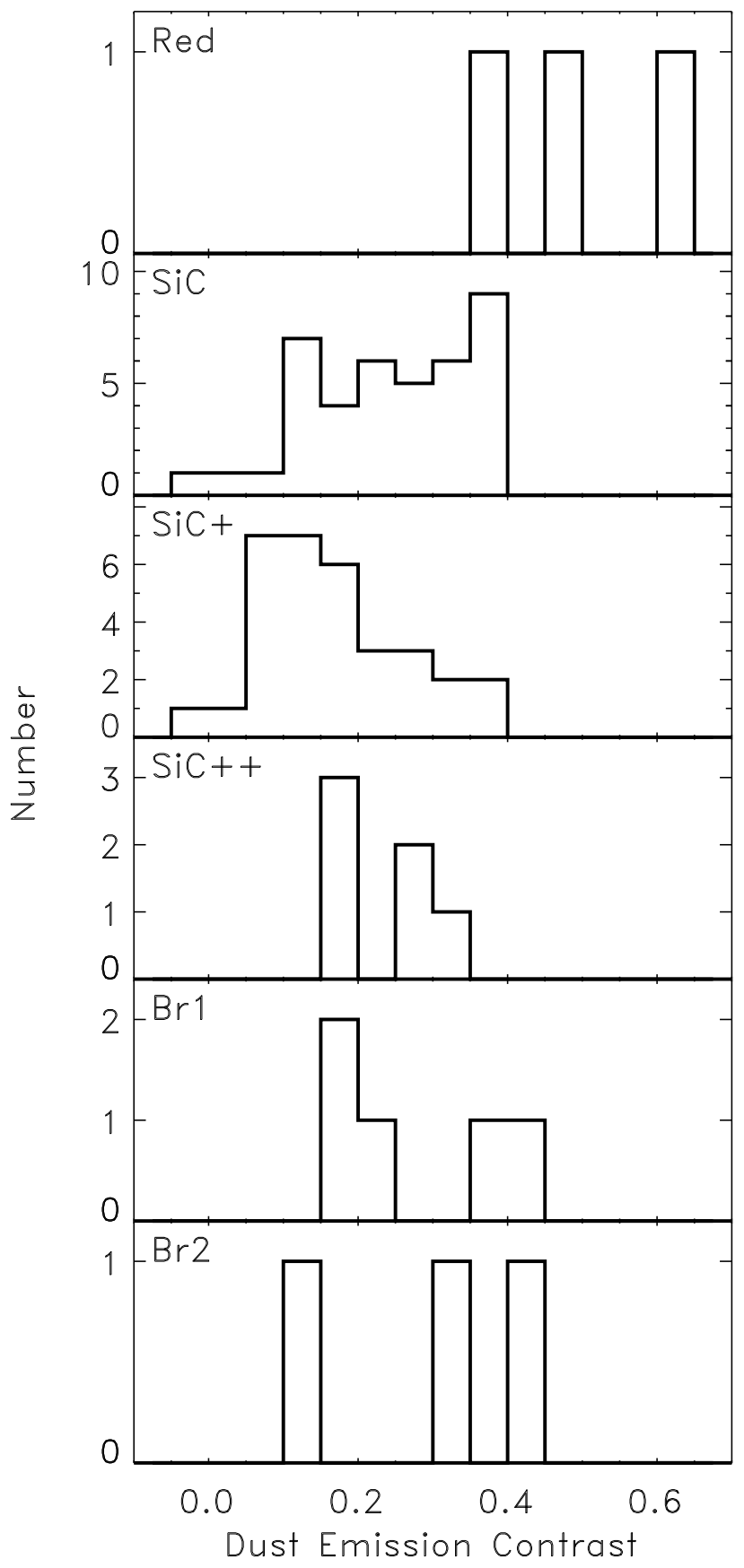

FIG. 4.-Distribution of dust emission contrasts for the six types of carbon-rich dust spectra identified.

TABLE 4

COMPARISON WITH THE AUTOCLASS II Classifications

\begin{tabular}{crrrrrrrrr}
\hline \hline & \multicolumn{8}{c}{ AUTOCLASS II } \\
\cline { 2 - 9 } Class & None & 0 & 1 & 3 & 4 & 5 & 6 & 9 & 11 \\
\hline $\operatorname{Red} \ldots \ldots \ldots \ldots$ & 1 & $\ldots$ & 1 & $\ldots$ & $\ldots$ & 1 & $\ldots$ & $\ldots$ & $\ldots$ \\
$\mathrm{SiC} \ldots \ldots \ldots \ldots$ & 5 & 26 & $\ldots$ & 7 & 2 & $\ldots$ & $\ldots$ & $\ldots$ & $\ldots$ \\
$\mathrm{SiC}+\ldots \ldots \ldots$ & 4 & 7 & $\ldots$ & 19 & 1 & $\ldots$ & $\ldots$ & 1 & $\ldots$ \\
$\mathrm{SiC}++\ldots \ldots$ & 1 & 1 & $\ldots$ & 4 & $\ldots$ & $\ldots$ & $\ldots$ & $\ldots$ & $\ldots$ \\
$\mathrm{Br} 1 \ldots \ldots \ldots \ldots$ & $\ldots$ & $\ldots$ & $\ldots$ & 4 & $\ldots$ & $\ldots$ & 1 & $\ldots$ & $\ldots$ \\
$\mathrm{Br} 2 \ldots \ldots \ldots \ldots$ & 1 & 1 & 1 & $\ldots$ & $\ldots$ & $\ldots$ & $\ldots$ & $\ldots$ & $\ldots$ \\
$\mathrm{SE} \ldots \ldots \ldots \ldots$ & $\ldots$ & $\ldots$ & $\ldots$ & 1 & $\ldots$ & $\ldots$ & $\ldots$ & $\ldots$ & 2 \\
$\mathrm{~N} \ldots \ldots \ldots \ldots$ & 2 & $\ldots$ & $\ldots$ & 1 & $\ldots$ & $\ldots$ & 1 & $\ldots$ & $\ldots$ \\
$\operatorname{Total} \ldots \ldots$ & 14 & 35 & 2 & 36 & 3 & 1 & 2 & 1 & 2 \\
\hline
\end{tabular}

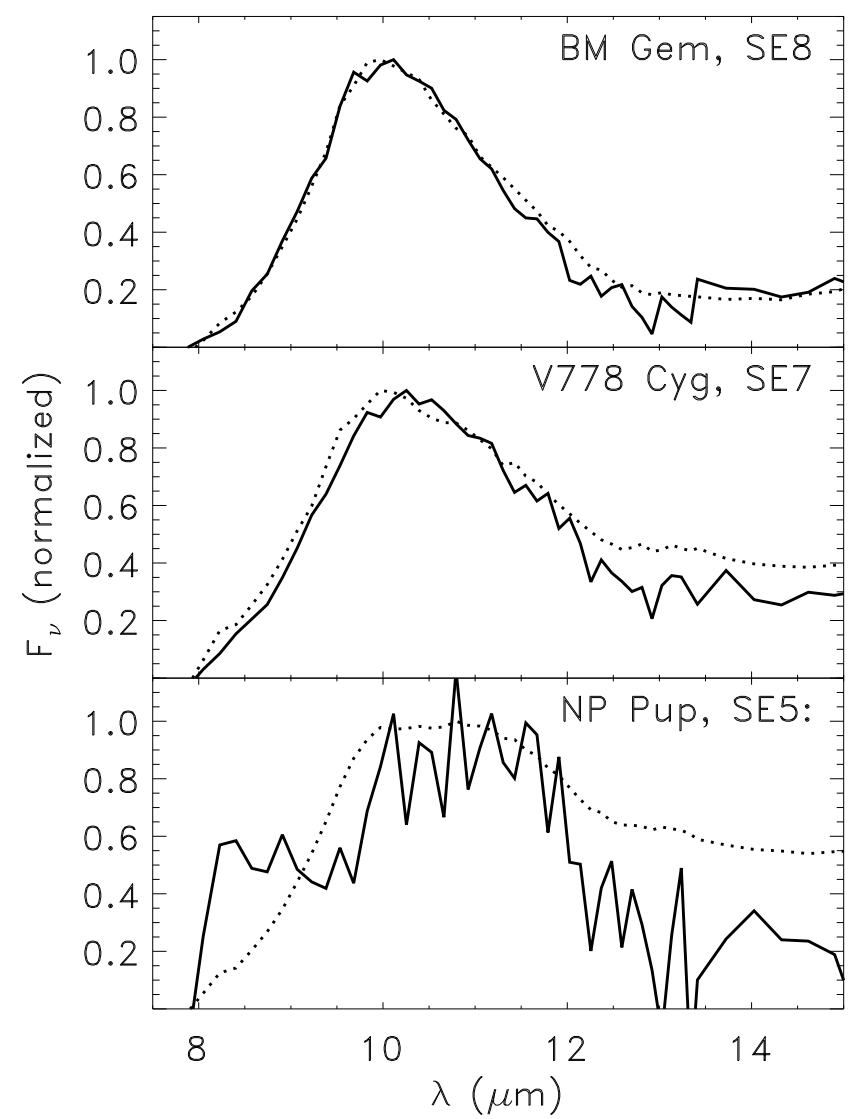

FIG. 5.-The two definite SE sources in our sample (top, middle) and the one suspect (bottom). The dotted spectrum in each panel is the sum of spectra of the corresponding SE class in the oxygen-rich AGB sample from SP2.

Figure 5 also illustrates the unusual spectrum of NP Pup, which is difficult to classify. The rise in emission from 8 to $10 \mu \mathrm{m}$ and the plateau from 10 to $12 \mu \mathrm{m}$ do not match the shape of any of the six carbon-rich classes. The closest fit to NP Pup is the average spectrum from SE5, but the SE5 spectrum shows substantially more emission beyond $12 \mu \mathrm{m}$ than NP Pup. Before identifying NP Pup as a silicate/ carbon star, we would recommend obtaining a higher quality mid-infrared spectrum to compare with the LRS data and a more careful look at its optical spectrum to make sure it is not an SC star.

Approximately $2 \%-3 \%$ of our sample of carbon stars show silicate emission. This percentage is very similar to the findings of Willems \& de Jong (1986) for a larger sample of 304 carbon stars. They found nine silicate/carbon stars, although Lloyd Evans (1990) later reduced this number to seven.

\section{DISCUSSION}

\subsection{The Carbon-rich Dust Sequence}

The properties of the different classes of carbon-rich dust lead us to identify the following carbon-rich dust sequence: $\mathrm{SiC}+\rightarrow \mathrm{SiC} \rightarrow$ Red.

As Table 5 shows, the percentage of Mira and SRa variables compared with $\mathrm{SRb}$ and $\mathrm{Lb}$ variables increases steadily along this sequence. Only $19 \%$ of the $\mathrm{SiC}+$ sources are Mira or SRa variables. This percentage increases to $63 \%$ of the $\mathrm{SiC}$ sources and $100 \%$ of the Red sources. Table 6 pre- 
TABLE 5

Distribution With Variability Class

\begin{tabular}{|c|c|c|c|c|c|}
\hline Class & Mira & $\mathrm{SRa}$ & $\mathrm{SRb}$ & $\mathrm{Lb}$ & Total \\
\hline Red. & 3 & & & 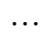 & 3 \\
\hline $\mathrm{SiC} \ldots \ldots \ldots$ & 22 & 3 & 10 & 5 & 40 \\
\hline $\mathrm{SiC}+\ldots \ldots \ldots$ & 5 & 1 & 16 & 10 & 32 \\
\hline $\mathrm{SiC}++\ldots \ldots$ & $\ldots$ & 1 & 3 & 2 & 6 \\
\hline $\operatorname{Br} 1 \ldots \ldots \ldots$ & 1 & 2 & 2 & $\ldots$ & 5 \\
\hline $\mathrm{Br} 2 \ldots \ldots \ldots$ & 1 & $\ldots$ & $\ldots$ & 2 & 3 \\
\hline SE $\ldots \ldots \ldots \ldots$ & ... & $\ldots$ & 1 & 2 & 3 \\
\hline $\mathrm{N} \ldots \ldots \ldots \ldots$ & & & 1 & 3 & 4 \\
\hline Total...... & 32 & 7 & 33 & 24 & 96 \\
\hline
\end{tabular}

sents, for each class, the average infrared colors, dust emission contrast, optical temperature index, and optical abundance index. Along the $\mathrm{SiC}+\rightarrow \mathrm{SiC} \rightarrow \mathrm{Red}$ sequence, the temperature index appears to increase. The Red sources are significantly cooler, while the $\mathrm{SiC}$ sources are marginally cooler on average than the $\mathrm{SiC}+$ sources. The steadily growing [12]-[25] colors and dust emission contrasts reveal that the dust shells become a dominant part of the total spectrum along the carbon-rich dust sequence.

The period of variability and the mass-loss rate also increase along the carbon-rich dust sequence (Table 7). For Mira and SRa variables, the mean period increases from $\mathrm{SiC}+$ to $\mathrm{SiC}$ to $\mathrm{Red}$. The periods of $\mathrm{SRb}$ variables also increase from $\mathrm{SiC}+$ to $\mathrm{SiC}$, although the distributions overlap significantly. The wider distributions probably result from the various pulsational modes observed in SRb variables. These results are consistent with the findings of Jones et al. (1990), who observed that the periods of stars increase monotonically as they become more heavily enshrouded in circumstellar dust. Ivezic \& Elitzur (1995) present mass-loss rates estimated from radiative transfer models and compare them with previously published deter- minations. In both sets of models, the mass-loss rates increase from $\mathrm{SiC}+$ to $\mathrm{SiC}$ to Red (although there is a difference of a factor of 2 between the two sets). The mass-loss rates in Table 7 are from Ivezic \& Elitzur (1995) and from sources listed by Netzer \& Elitzur (1993), except for the $\mathrm{SiC}++$ sources, which are from models by Jura (1986) and Loup et al. (1993).

In summary, along the carbon-rich dust sequence (1) the stars become cooler; (2) they pulsate more regularly, with stronger amplitude, and with longer periods; (3) they become more heavily enshrouded in dust as the mass-loss rate increases; (4) they show a decreasing contribution from the 8.5-9.0 $\mu \mathrm{m}$ emission feature; and (5) the dust continuum produced by amorphous carbon grows steadily in strength.

These results strongly support the findings of Willems (1988a, 1988b) and Willems \& de Jong (1988), who noted the difference in spectral properties between carbon-rich Mira variables on the one hand and carbon-rich semiregular and irregular variables on the other. They argued that the strong and steady pulsations of the Mira variables would drive a higher mass-loss rate, which would lead to thicker, redder shells showing the $\mathrm{SiC}$ component and the red dust continuum. Semiregular and irregular variables, however, pulsate with smaller amplitudes; the resulting lower mass-loss rates would produce thinner shells showing contributions from $\mathrm{SiC}$ dust and the additional feature at $\sim 8.5-9.0 \mu \mathrm{m}$, but not the dust continuum present in Mira variables. These authors assumed that the difference in mass-loss rate resulted from a shift from the overtone mode (for semiregular and irregular variables) to the fundamental mode (for Mira variables), and this assumption has received support from recent work (Wood 1995; Wood \& Sebo 1996; van Belle et al. 1996).

We note that a straightforward evolutionary scheme from irregular to Mira variable could be interrupted by thermal pulses. Willems \& de Jong (1988) have suggested

TABLE 6

INFRARED AND OPTICAL PROPERTIES

\begin{tabular}{ccccc}
\hline \hline Class & {$[12]-[25]$} & \multicolumn{1}{c}{ DEC $^{\mathrm{a}}$} & Temperature Index $^{\mathrm{b}}$ & Abundance Index $^{\mathrm{b}}$ \\
\hline Red $\ldots \ldots \ldots .$. & $0.72 \pm 0.10$ & $0.48 \pm 0.13$ & $7.8 \pm 1.3$ & $4.2 \pm 0.8$ \\
$\mathrm{SiC} \ldots \ldots \ldots$. & $0.35 \pm 0.16$ & $0.24 \pm 0.11$ & $5.9 \pm 1.5$ & $3.0 \pm 1.0$ \\
$\mathrm{SiC}+\ldots \ldots \ldots$ & $0.25 \pm 0.14$ & $0.16 \pm 0.11$ & $5.6 \pm 0.9$ & $3.9 \pm 1.0$ \\
$\mathrm{SiC}+\ldots \ldots \ldots$ & $0.32 \pm 0.26$ & $0.24 \pm 0.07$ & $5.0 \pm 1.0$ & $5.3 \pm 0.7$ \\
$\mathrm{Br} 1 \ldots \ldots \ldots$. & $0.34 \pm 0.10$ & $0.27 \pm 0.12$ & $7.3 \pm 1.3$ & $2.8 \pm 0.5$ \\
$\mathrm{Br} 2 \ldots \ldots \ldots \ldots$ & $0.37 \pm 0.20$ & $0.28 \pm 0.15$ & $7.2 \pm 1.0$ & $2.7 \pm 0.6$ \\
$\mathrm{SE} \ldots \ldots \ldots \ldots$ & $0.79 \pm 0.31$ & $1.25 \pm 0.98$ & $5.3 \pm 1.5$ & $3.7 \pm 1.5$ \\
$\mathrm{~N} \ldots \ldots \ldots \ldots$ & $0.05 \pm 0.08$ & $-0.03 \pm 0.06$ & $5.8 \pm 1.3$ & $3.8 \pm 1.3$ \\
\hline
\end{tabular}

${ }^{a}$ Dust emission contrast.

b From the optical spectral class.

TABLE 7

Periods AND Mass-Loss Rates

\begin{tabular}{|c|c|c|c|c|}
\hline \multirow[b]{2}{*}{ ClaAss } & \multicolumn{2}{|c|}{ PERIOD (days) } & \multicolumn{2}{|c|}{$\begin{array}{c}\langle\dot{M}\rangle \\
\left(10^{-6} M_{\odot} \mathrm{yr}^{-1}\right)\end{array}$} \\
\hline & Mira and SRa & $\mathrm{SRb}$ & Quoted $^{\mathrm{a}}$ & Derived $^{\mathrm{b}}$ \\
\hline Red & $590 \pm 60$ & & 28 & 5.4 \\
\hline $\mathrm{SiC} \ldots \ldots \ldots . .$. & $460 \pm 60$ & $290 \pm 150$ & 2.4 & 1.3 \\
\hline $\mathrm{SiC}+\ldots \ldots \ldots$ & $410 \pm 80$ & $240 \pm 110$ & 1.0 & 0.4 \\
\hline $\mathrm{SiC}++\ldots \ldots$ & $370 \pm 0$ & $350 \pm 150$ & 1.8 & $\ldots$ \\
\hline
\end{tabular}

${ }^{\text {a }}$ Quoted by Ivezic \& Elitzur 1995; references given by Netzer \& Elitzur 1993

${ }^{\text {b }}$ From models of Ivezic \& Elitzur 1995. 
that semiregular and irregular variables may result when a thermal pulse upsets the regular pulsational behavior of a Mira variable. The effect would be several episodes of mass loss on timescales corresponding to the intervals between thermal pulses, which can vary from thousands to hundreds of thousands of years (Iben \& Renzini 1983).

The abundance indices (Table 6) present a more confusing picture. We expect that as a star evolves up the AGB, repeated dredge-ups of carbon-rich material should produce a corresponding increase in $\mathrm{C} / \mathrm{O}$ abundance. However, a comparison of the two classes with statistically significant populations shows that the $\mathrm{SiC}+$ sources exhibit a somewhat higher carbon abundance index than the $\mathrm{SiC}$ sources $(3.9 \pm 1.0$ as compared with $3.0 \pm 1.0)$. The same marginal trend appears in the $\mathrm{C} / \mathrm{O}$ abundance measurements by Lambert et al. (1986). The average C/O ratio for $13 \mathrm{SiC}+$ stars is $1.13 \pm 0.09$ as compared with $1.06 \pm 0.03$ for the six $\mathrm{SiC}$ sources in their sample.

\subsection{The $\mathrm{SiC}++$ Class}

In preliminary discussions of this work (Sloan, LittleMarenin, \& Price 1996; Little-Marenin, Sloan, \& Price 1997), we assigned the $\mathrm{SiC}++$ class as the first step of the carbon-rich dust sequence, prior to $\mathrm{SiC}+$ sources, based primarily on the strength of the secondary emission feature at $\sim 8.5-9.0 \mu \mathrm{m}$, and to a lesser extent on the smaller temperature indices (warmer central stars) and the fact that all but one of the six $\mathrm{SiC}++$ sources is an $\mathrm{SRb}$ or $\mathrm{Lb}$ variable. Here, we abandon this assignment because the red [12][25] colors, large dust emission contrasts, and unusually high carbon abundances do not fit into the carbon-rich dust sequence.

The $\mathrm{C} / \mathrm{O}$ ratios for the four $\mathrm{SiC}++$ stars analyzed by Lambert et al. (1986) are 1.2 for TW Oph, 1.29 for T Lyr, 1.34 for $\mathrm{R} \mathrm{Scl}$, and 1.76 (the largest $\mathrm{C} / \mathrm{O}$ ratio in their sample of carbon stars) for VX And. The average $\mathrm{C} / \mathrm{O}$ ratio of 1.40 is significantly higher than for the $\mathrm{SiC}+$ and $\mathrm{SiC}$ stars, and continues the trend of higher $\mathrm{C} / \mathrm{O}$ ratios as the relative strength of the feature at $\sim 8.5-9.0 \mu \mathrm{m}$ grows. Figure 3 shows an apparent drop in the mean $\mathrm{SiC}++$ spectrum below zero in the $13-15 \mu \mathrm{m}$ range, possibly a result of photospheric absorption from $\mathrm{C}_{2} \mathrm{H}_{2}$ or $\mathrm{HCN}$ (Willems 1988a). Among the $\mathrm{SiC}++$ sources, the $\mathrm{C} / \mathrm{O}$ ratio generally correlates with the strength of the absorption at $\sim 13-15$ $\mu \mathrm{m}$ and the strength of the feature at $\sim 8.5-9.0 \mu \mathrm{m}$ (relative to the $11.2 \mu \mathrm{m}$ feature).

\subsection{J Stars}

Bouigue (1954) first defined the relatively rare J-type carbon stars as carbon stars with unusually strong isotopic ${ }^{13} \mathrm{CN}$ bands at $6260 \AA \AA$. The $\mathrm{J}$ stars show no enhancements in s-process elements and, based on their lower luminosities compared with other carbon stars, are probably not AGB stars. While J stars represent only $10 \%$ of our carbon stars (nine of 96), they represent two of the six $\mathrm{SiC}++$ sources. Information on the ${ }^{12} \mathrm{C} /{ }^{13} \mathrm{C}$ ratio for one of the remaining four $\mathrm{SiC}++$ sources (namely, W Pic) is not available, so the fraction may be higher.

The J stars may be the key to explaining the unusual properties of the $\mathrm{SiC}++$ sources. Of the nine $\mathrm{J}$ stars in our sample, most are unusual in one way or another. In addition to the two $\mathrm{SiC}++$ sources, one is among the four nearly naked sources, another is among the five Broad 1 sources, and both confirmed SE sources are $\mathbf{J}$ stars. While three $\mathbf{J}$ stars are among the more normal $\mathrm{SiC}+$ class, two of these show possible absorption at $\sim 14 \mu \mathrm{m}$, as is seen in the $\mathrm{SiC}++$ spectra. One of these was tentatively assigned to the $\mathrm{SiC}++$ group early in the classification process.

Lorenz-Martins \& Lefèvre $(1993,1994)$ and LorenzMartins (1996) have estimated the ratio of amorphous carbon to silicon carbide in carbon-rich dust shells by fitting radiative transfer models to spectral energy distributions and LRS data. They find that as the optical depth of the shell (and therefore the mass-loss rate from the star) increases, the fraction of amorphous carbon increases, from $80 \%-90 \%$ for optically thin shells to $\sim 95 \%$ for optically thick shells. The fraction of SiC drops to zero for sources with $\dot{M}>10^{-4} M_{\odot} \mathrm{yr}^{-1}$ (Groenewegen 1995). The J stars do not fit into this sequence. The ratio of amorphous carbon to $\mathrm{SiC}$ in shells around $\mathbf{J}$ stars excedes the ratio expected for the relatively low optical depth of their shells.

Willems \& de Jong (1986) first suggested that the J stars might be the sole source of silicate/carbon stars, a hypothesis confirmed by Lambert, Hinkle, \& Smith (1990) and Lloyd Evans (1990). However, the suggestion of Willems \& de Jong (1988) that the silicate/carbon stars and the J stars represent a transition from oxygen-rich to carbon-rich photosphere has proven less successful. They suggested that when a thermal pulse and subsequent dredge-up convert a stellar photosphere to carbon-rich, the circumstellar shell of oxygen-rich dust would dissipate slowly enough to explain the silicate/carbon stars. Lambert et al. (1990) and Lloyd Evans (1990) argued that the oxygen-rich dust shell should grow spectrally invisible within $\sim 50$ years - too brief a period to account for the number of observed silicate/ carbon stars. In addition, Lambert et al. (1990) pointed out that this hypothesis does not explain the unusually high abundance of ${ }^{13} \mathrm{C}$ that defines the $\mathrm{J}$ stars.

Instead, Lambert et al. (1990) and Lloyd Evans (1990) argue that the silicate/carbon stars result from the storage of oxygen-rich material lost prior to the AGB phase in a disk around a companion star. They speculate that this mass loss may occur during the helium core flash. Most stars would retain oxygen-rich photospheres, so oxygenrich dust around a companion would not attract undue attention. However, for reasons that are not clear, some stars would be converted during or soon after the helium core flash to carbon stars. In the standard scenario, this conversion does not occur until the third dredge-up on the asymptotic giant branch, when by-products of the $3 \alpha$ process rich in s-process elements and ${ }^{12} \mathrm{C}$ (but not ${ }^{13} \mathrm{C}$ ) are convected to the surface (Iben \& Renzini 1983).

Lambert et al. (1990) suggested that J stars, which have an overabundance of ${ }^{13} \mathrm{C}$ but virtually no excess in $s$ process elements, evolve on a sequence parallel to the normal $\mathrm{M} \rightarrow \mathrm{S} \rightarrow \mathrm{C}$ sequence on the AGB. The J stars may evolve from the early R stars, which, while already carbonrich, are not luminous enough to have reached the AGB and may instead be on the helium-burning main sequence. The early $R$ stars also show enhancements in ${ }^{13} \mathrm{C}$ and have no s-process enhancements (Dominy 1984). In the alternative scenario, the $\mathbf{J}$ stars that appear as silicate/carbon stars are the rare cases in which a companion is available to store oxygen-rich dust formed prior to the conversion to carbon star. Eventually, mass loss from the $\mathbf{J}$ star may produce enough carbon-rich dust to mask the oxygen-rich disk.

While the silicate/carbon stars represent an unusual evolutionary path for $\mathbf{J}$ stars, their existence requires that the $\mathbf{J}$ 
stars do not follow the same evolutionary path as the rest of the carbon stars. Lorenz-Martins (1996) also suggests a different sequence for all $\mathbf{J}$ stars because of the unusually high amorphous carbon/silicon carbide ratios for the optical depth of their shells. We have already noted that most of the $\mathbf{J}$ stars in our sample show unusual dust spectra compared with the more normal carbon stars. An unusual dust chemistry would arise naturally from the unusual abundances in the stellar photosphere.

\subsection{The Broad Carbon-rich Spectra}

The broad classes $(\mathrm{Br} 1$ and $\mathrm{Br} 2)$ show similar color distributions to the $\mathrm{SiC}$ class, but they are associated with much cooler central stars. This difference supports our suspicion that the stars showing broad $\mathrm{SiC}$ emission represent a physically different class of objects. Unfortunately, little else can be concluded about these sources from our sample.

\subsection{The $9.0 \mu \mathrm{m}$ Feature}

The identity of the carrier of the secondary emission feature at $\sim 8.5-9.0 \mu \mathrm{m}$ remains much less certain than the other carbon-rich dust features discussed in this paper. A careful examination of Figure 3 reveals that this feature peaks closer to $9.0 \mu \mathrm{m}$ than 8.6 or $8.7 \mu \mathrm{m}$, as often described in other papers. We attribute this difference to two factors. First, using the correction factors of Cohen et al. (1992) removes a spectral artifact that peaks at $\sim 8.3 \mu \mathrm{m}$. Second, other investigators have usually examined the secondary emission feature without removing the underlying blue continuum. Both these factors will shift the feature from $9.0 \mu \mathrm{m}$ to shorter wavelengths.

The $9.0 \mu \mathrm{m}$ feature is very prominent in the $\mathrm{SiC}++$ sources. Because these sources have such high $\mathrm{C} / \mathrm{O}$ ratios, we suspect that photospheric absorption from carbon-rich molecules such as $\mathrm{C}_{2} \mathrm{H}_{2}$ and $\mathrm{HCN} \sim 7-8 \mu \mathrm{m}$ is causing us to underestimate the stellar continuum. Spectra of $\mathrm{SiC}++$ sources covering the 4-8 $\mu \mathrm{m}$ wavelength range obtained from the Kuiper Airborne Observatory (KAO; J. Bregman 1997, private communication) and from the Japanese Infrared Telescope in Space (IRTS; I. Yamamura 1997, private communication) show strong photospheric absorption bands centered at 5.2 and $7.5 \mu \mathrm{m}$. The level of the continuum under the 9.0 and $11.2 \mu$ m features depends on how the LRS data are joined to these spectra, making it very difficult to state conclusively how much of the $9 \mu \mathrm{m}$ emission feature is actually due to absorption at neighboring wavelengths. Hopefully, published spectra from IRTS and the Infrared Space Observatory (ISO) will provide the answer.

In spectra of $\mathrm{SiC}+$ and other dust classes, the photospheric absorption feature at $\sim 7.5 \mu \mathrm{m}$ is much weaker. In published spectra from IRTS, two SiC + sources, RY Dra and U Cam, show almost no absorption at $7.5 \mu \mathrm{m}$ compared with $\mathrm{T}$ Lyr, an $\mathrm{SiC}++$ source (Yamamura et al. 1997a, 1997b). Figure 6 illustrates two of the KAO spectra (obtained through a circularly variable filter wheel). We have normalized the KAO data to match the LRS data at 8 $\mu \mathrm{m}$ and fitted blackbodies through the continuum. In these spectra, the majority of the emission from the $9.0 \mu \mathrm{m}$ feature appears to be too strong to be attributed to an artifact from photospheric absorption. We therefore continue our discussion with the assumption that in the $\mathrm{SiC}+$ and $\mathrm{Br} 1$ spectra, the $9.0 \mu \mathrm{m}$ feature is truly a dust emission feature.

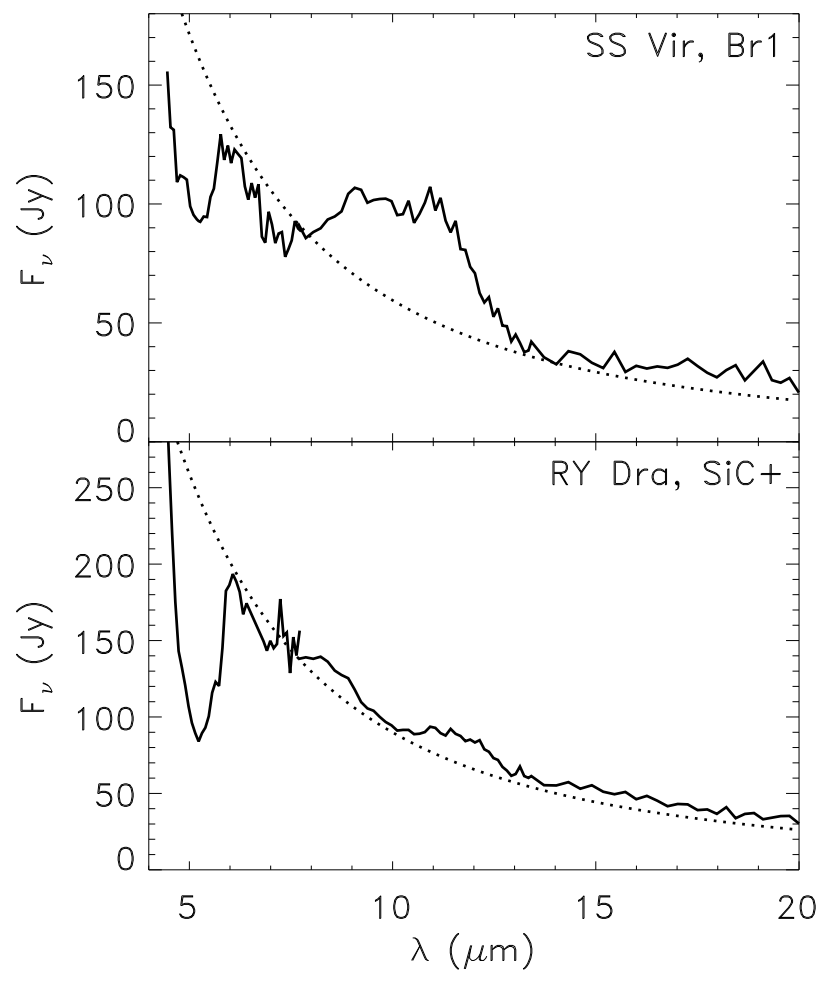

FIG. 6.-Spectra from the KAO of two carbon stars (4-8 $\mu \mathrm{m})$, normalized to match the LRS spectrum $(8-20 \mu \mathrm{m})$. The dotted line is a $2400 \mathrm{~K}$ Planck function to illustrate the probable photospheric continuum level. Absorption features are probably photospheric; emission features are probably from the dust shell.

The strength of the $9.0 \mu \mathrm{m}$ feature and the cool amorphous carbon component are completely anticorrelated. In optically thin shells, where the emission is dominated by the warmer temperatures and higher densities of the dust formation zone, we see the $9.0 \mu \mathrm{m}$ feature. However, when the shell is thick enough to hide the dust formation zone and move the dust "photosphere" out to much cooler temperatures, the $9.0 \mu \mathrm{m}$ feature disappears and is replaced by the amorphous carbon component.

Goebel et al. (1995) propose a carrier most consistent with the anticorrelation of the $9.0 \mu \mathrm{m}$ feature and the cool continuum: a precursor to graphite and amorphous carbon. They suggest further that it might consist of a material described as a-C:H (hydrogenated hard carbon; Dischler, Bubenzer, \& Koidl 1982, 1983). This material is essentially a hydrocarbon mix with the spectral properties of polycyclic aromatic hydrocarbons (PAHs), which produce the wellknown PAH spectrum with features at $\sim 7.7,8.6$, and 11.3 $\mu \mathrm{m}$. The $8.6 \mu \mathrm{m}$ feature arises from an out-of-plane $\mathrm{C}-\mathrm{H}$ bend and usually appears as a shoulder on the much larger $7.7 \mu \mathrm{m}$ emission feature. The wavelength shift from 8.6 to $9.0 \mu \mathrm{m}$ could conceivably result from an unusual size or shape distribution, but the absence of the $7.7 \mu \mathrm{m}$ feature in the carbon star spectra is much more difficult to explain.

A related hypothesis is that the $9.0 \mu \mathrm{m}$ feature arises from a $\mathrm{PAH}$ precursor, perhaps from a $\mathrm{C}-\mathrm{O}$ bond. Morterra \& Low (1983) produced PAH-like material by charring cellulose. Their uncharred samples show an emission feature near 9.3-9.4 $\mu \mathrm{m}$ while in the charred samples this feature has vanished, to be replaced with the PAH features at $\sim 7.7$, 8.6, and $11.3 \mu \mathrm{m}$. The energies of the $9.0 \mu \mathrm{m}$ feature 
observed in carbon stars and the feature in the cellulose samples suggest a $\mathrm{C}-\mathrm{O}$ bond within a carbon matrix (Nakanishi \& Solomon 1977). Thus the $9.0 \mu \mathrm{m}$ feature could arise from a $\mathrm{C}-\mathrm{O}$ bond in a carbonate material. The $\mathrm{C}-\mathrm{O}$ bonds could originate in chemical reactions with the $\mathrm{CO}$ gas in the outflows. As the carbon-rich material anneals, these relatively fragile bonds will break, releasing the oxygen for recombination as $\mathrm{CO}$ while the carbon matrix grows into amorphous carbon particles or graphite.

\section{CONCLUSION}

Our classification of a sample of nearly 100 bright carbon stars shows that most sources produce a spectrum with an $\mathrm{SiC}$ emission feature at $11.2 \mu \mathrm{m}$, accompanied by either a cool dust continuum attributed to amorphous carbon or graphite or a secondary emission feature at $9.0 \mu \mathrm{m}$ of unknown origin. These two spectral components are anticorrelated and represent a natural means of ordering the spectra from carbon stars along the carbon-rich dust sequence. Tracing the sequence in the direction of a decreasing contribution from the $9.0 \mu \mathrm{m}$ feature and an increasing contribution from amorphous carbon reveals several trends. The central star grows cooler, the dust shell increases in spectral contrast in the infrared and grows redder, the mass-loss rate increases, the fraction of Mira variables compared with semiregular and irregular variables increases, and the period of variability increases. Thus the $9.0 \mu \mathrm{m}$ feature is visible only in dust shells that are optically thinner, where emission from the dust formation zone dominates the total dust spectrum. Amorphous carbon emission is most noticeable in thicker shells, where the outer parts of the dust shell dominate the emission from the inner regions.

The carbon-rich dust sequence could represent an evolutionary sequence traced by stars evolving from irregular pulsators to Mira variables, whose stronger, steadier pulsa- tions drive higher mass-loss rates and produce thicker shells.

The $\mathrm{SiC}++$ spectra do not fall on the carbon-rich dust sequence. Their unusual infrared spectral properties may arise from photospheric absorption as much as from circumstellar dust emission. This hypothesis is consistent with the high $\mathrm{C} / \mathrm{O}$ ratios in these stars, which would lead to very strong photospheric absorption bands from carbon-rich molecules. We speculate that the $\mathrm{SiC}++$ sources may lie on a different evolutionary sequence that may include the $\mathbf{J}$ stars, and possibly the silicate/carbon stars as well.

While the case for the $9.0 \mu \mathrm{m}$ feature as a precursor to amorphous carbon has some merit, the low resolution and limited wavelength range of the LRS data make a firm identification of carriers of small emission features like the 9.0 $\mu \mathrm{m}$ feature difficult. With new data from ISO and IRTS, the origin of the $9.0 \mu \mathrm{m}$ feature should become clearer. These satellites should also improve our understanding of the infrared photospheric continuum in carbon stars, an important step in improving the classification scheme developed here.

We would like to thank J. Bregman, J. Goebel, M. Bernstein, and L. Allamandola for their useful comments on the manuscript, and for sharing unpublished astronomical and laboratory data with us. We are especially grateful to I. Yamamura for providing us with preliminary spectra from IRTS. This research made use of the LRS database maintained at the University of Calgary, the SIMBAD database at Strasbourg, and data and software distributed by the NASA Astronomical Data Center. G. C. S. received support from the Phillips Laboratory Scholar program and the National Research Council, and would also like to thank the Physics Department of the University of New England in Armidale, Australia, for graciously hosting him during the writing of this paper.

\section{REFERENCES}

Atlas of Low Resolution IRAS Spectra. 1986, IRAS Science Team, prepared by F. M. Olnon \& E. Raimond (A\&AS, 65, 607)

Baron, Y., de Muizon, M., Papoular, R., \& Pégourié, B. 1987, A\&A, 186, 271

Bouigue, R. 1954, Ann. d'Astrophys., 17, 104

Chan, S. J., \& Kwok, S. 1988, ApJ, 334, 362

. 1990, A\&A, 237, 354

Cheeseman, P., Stutz, J., Self, M., Taylor, W., Goebel, J., Volk, K., \&

Walker, H. 1989, Automatic Classification of Spectra from the Infrared Astronomical Satellite (IRAS) (NASA RP-1217) (Washington: NASA)

Cohen, M. 1984, MNRAS, 206, 137

Cohen, M., Walker, R. G., \& Witteborn, F. C. 1992, AJ, 104, 2030

Dischler, B., Bubenzer, A., \& Koidl, P. 1982, Appl. Phys. Lett., 42, 636

. 1983, Solid State Commun., 48, 105

Dominy, J. F. 1984, ApJS, 55, 27

Gilman, R. C. 1969 , ApJ, 155, L185

Goebel, J. H., et al. 1980, ApJ, 235, 104

Goebel, J. H., Cheeseman, P., \& Gerbault, F. 1995, ApJ, 449, 246

Goebel, J. H., Volk, K., Walker, H., Gerbault, F., Cheeseman, P., Self, M.,

Stutz, J., \& Taylor, W. 1989, A\&A, 222, L5

Griffin, I. P. 1990, MNRAS, 247, 591

Groenewegen, M. A. T. 1995, A\&A, 293, 463

. 1997, A\&A, 317, 503

Habing, H. J. 1990, in From Miras to Planetary Nebulae, ed. M. O. Mennessier \& A. Omont (Gif-sur-Yvette: Ed. Frontières), 16

Hackwell, J. A. 1972, A\&A, 21, 239

Hearn, A. G. 1990, in From Miras to Planetary Nebulae, ed. M. O. Mennessier \& A. Omont (Gif-sur-Yvette: Ed. Frontières), 121

Iben, I., \& Renzini, A. 1983, ARA\&A, 21, 271

IRAS Point Source Catalog, Version 2. 1988, Joint IRAS Science Working Group (Washington: GPO) (PSC)

Ivezic, Z., \& Elitzur, M. 1995, ApJ, 445, 415

Jones, T. J., Bryja, C. O., Gehrz, R. D., Harrison, T. E., Johnson, J. J.,

Klebe, D. I., \& Lawrence, G. F. 1990, ApJS, 74, 785

Jura, M. 1986, ApJ, 303, 327
Kholopov, P. N., et al. 1985-1988, General Catalogue of Variable Stars (4th ed.; Moscow: Nauka) (GCVS)

Lambert, D. L., Gustaffson, B., Eriksson, K., \& Hinkle, K. H. 1986, ApJS, 62,373

Lambert, D. L., Hinkle, K. H., \& Smith, V. V. 1990, AJ, 99, 1612

Little-Marenin, I. R., \& Little, S. J. 1988, ApJ, 333, 305

. 1990, ApJ, 99, 1173

Little-Marenin, I. R., Sloan, G. C., \& Price, S. D. 1997, in IAU Symp. 177, The Carbon Star Phenomenon, ed. R. F. Wing (Dordrecht: Kluwer), in press

Lloyd Evans, T. 1990, MNRAS, 243, 336

Lorenz-Martins, S. 1996, A\&A, 314, 209

Lorenz-Martins, S., \& Lefèvre, J. 1993, A\&A, 280, 567

. 1994, A\&A, 291, 831

Loup, C., Forveille, T., Omont, A., \& Paul, J. F. 1993, A\&AS, 99, 291

Martin, P. G., \& Rogers, C. 1987, ApJ, 322, 374

Mikami, T. 1975, PASJ, 27, 445

Morterra, C., \& Low, M. J. D. 1983, Carbon, 21, 283

Nakanishi, K., \& Solomon, P. H. 1977, Infrared Absorption Spectroscopy (2d ed.; San Francisco: Holden-Day)

Netzer, N., \& Elitzur, M. 1993, ApJ, 410, 701

Onaka, T., de Jong, T., \& Willems, F. J. 1989, A\&A, 218, 169

Papoular, R. 1988, A\&A, 204, 138

Pégourié, B. 1987, A\&A, 194, 335

Simpson, J. P. 1991, ApJ, 368, 570

Sloan, G. C., \& Egan, M. P. 1995, ApJ, 444, 452

Sloan, G. C., Little-Marenin, I. R., \& Price, S. D. 1996, in From Stardust to Planetesimals: Contributed Papers, ed. M. E. Kress, A. G. G. M. Tielens, $\&$ Y. Pendleton (NASA CP-3343) (Moffett Field: NASA), 65

Sloan, G. C., \& Price, S. D. 1995, ApJ, 451, 758 (SP1) 1997, in preparation (SP2)

Speck, A. K., Barlow, M. J., \& Skinner, C. J. 1996, in From Stardust to Planetesimals: Contributed Papers, ed. M. E. Kress, A. G. G. M. Tielens, \& Y. Pendleton (NASA CP-3343) (Moffett Field: NASA), 61

. 1997, MNRAS, 288, 431 
Treffers R., \& Cohen, M. 1974, ApJ, 188, 545

van Belle, G. T., Dyck, H. M., Benson, J. A., \& Lacasse, M. G. 1996, AJ, 112,2147

Vardya, M., de Jong, T., \& Willems, F. J. 1986, ApJ, 304, L29

Willems, F. J. 1988a, A\&A, 203, 51 . 1988b, A\&A, 203, 65

Willems, F. J., \& de Jong, T. 1986, ApJ, 309, L39 1988, A\&A, 196, 173

Wood, P. R. 1995, in IAU Colloq. 155, Astrophysical Applications of Stellar Pulsations, ed. R. S. Stobie \& P. A. Whitelock (ASP Conf. Ser. 83) (San Francisco: ASP), 127
Wood, P. R., \& Sebo, K. M. 1996, MNRAS, 282, 958

Yamamura, I., et al. 1997a, in ASP Conf. Ser. 124, Diffuse Infrared Radiation and the IRTS, ed. T. L. Roellig, T. Matsumoto, \& H. Okuda (San Francisco: ASP), in press

Yamamura, I., Onaka, T., Tanabé, T., Roellig, T. L., \& Yuen, L. 1997b, PASJ, in press 